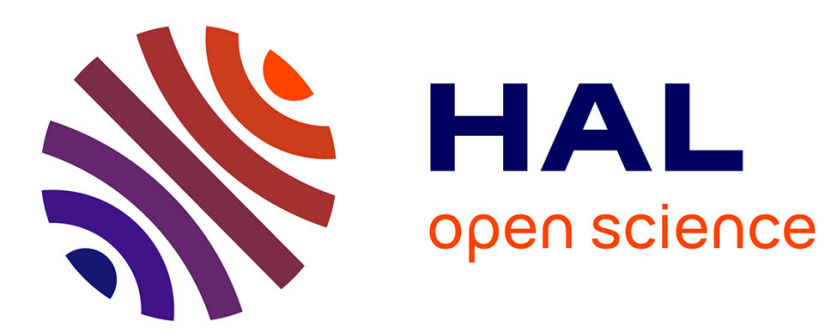

\title{
The determinants of efficiency and productivity in European railways
}

Antonio Couto, Daniel J. Graham

\section{To cite this version:}

Antonio Couto, Daniel J. Graham. The determinants of efficiency and productivity in European railways. Applied Economics, 2009, 41 (22), pp.2827-2851. 10.1080/00036840801949782 . hal-00582206

\section{HAL Id: hal-00582206 \\ https://hal.science/hal-00582206}

Submitted on 1 Apr 2011

HAL is a multi-disciplinary open access archive for the deposit and dissemination of scientific research documents, whether they are published or not. The documents may come from teaching and research institutions in France or abroad, or from public or private research centers.
L'archive ouverte pluridisciplinaire HAL, est destinée au dépôt et à la diffusion de documents scientifiques de niveau recherche, publiés ou non, émanant des établissements d'enseignement et de recherche français ou étrangers, des laboratoires publics ou privés. 


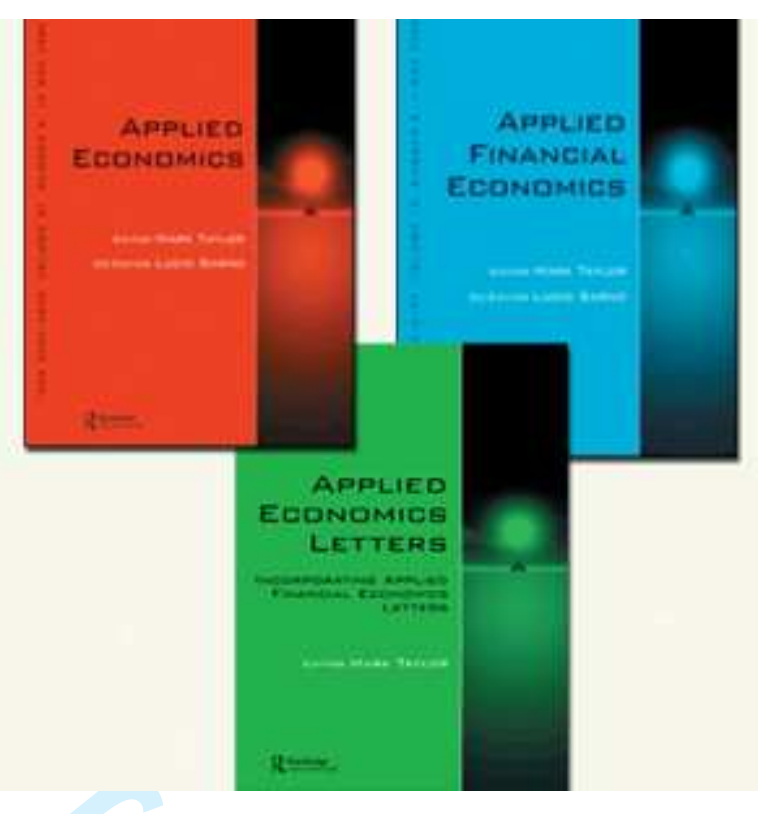

The determinants of efficiency and productivity in European railways

\begin{tabular}{|c|c|}
\hline Journal: & Applied Economics \\
\hline Manuscript ID: & APE-06-0800 \\
\hline Journal Selection: & Applied Economics \\
\hline $\begin{array}{r}\text { Date Submitted by the } \\
\text { Author: }\end{array}$ & 18-Dec-2006 \\
\hline Complete List of Authors: & $\begin{array}{l}\text { Couto, Antonio; University of Porto, Research Centre for Territory, } \\
\text { Transport and Environment } \\
\text { Graham, Daniel; Imperial College London, Centre for Transport } \\
\text { Studies }\end{array}$ \\
\hline JEL Code: & $\begin{array}{l}\text { R41 - Transportation: Demand } \mid \text { Supply } \mid \text { Congestion } \mid \text { Safety and } \\
\text { Accidents < R4 - Transportation Systems < R - Urban, Rural, and } \\
\text { Regional Economics, D24 - Production } \mid \text { Capital and Total Factor } \\
\text { Productivity|Capacity < D2 - Production and Organizations < D - } \\
\text { Microeconomics }\end{array}$ \\
\hline Keywords: & \\
\hline
\end{tabular}




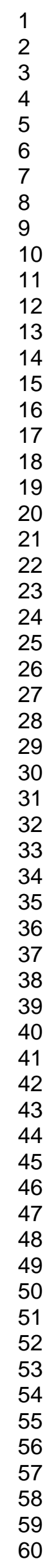

\section{SCHOLARONE ${ }^{m}$ \\ Manuscripts}

7

21

23

24

26

27

29

30

32

33

34

36

37

38

41

42

43

45

46

47

48

49

51

52

53

54

55

57

58

59

60 


\title{
The determinants of efficiency and productivity in European railways
}

\author{
Antonio Couto ${ }^{\dagger}$ \\ University of Porto \\ Daniel J Graham ${ }^{\dagger \dagger}$ \\ Imperial College London
}

\begin{abstract}
This paper evaluates the economic performance of European railways over the period 1972 to 1999 . The cost structure of the railway industry is analysed using a stochastic frontier approach estimated within the framework of a translog cost system. The results confirm that European railways experience significant cost increases due to inefficient behaviour. In contrast to previous studies, however, the estimates indicate a much greater role for allocative rather than technical inefficiency. Overall, inefficiencies can essentially be explained by the supply of excess capacity and by the over-employment of labour inputs. Regarding productivity, it appears to be technological progress, and not levels of efficiency or scale economies, that provide the most convincing explanation for variance in growth rates within the sample.
\end{abstract}

Key words: Cost function, Stochastic Frontier, Efficiency, Productivity.

JEL Classification: R41, D24.

\footnotetext{
${ }^{\dagger}$ Research Centre for Territory, Transports and Environment, FEUP, Rua Dr. Roberto Frias 4200-465 Porto-Portugal. Email: fcouto@fe.up.pt, Tel: +351 2250821 10, Fax: +351 225081902.

${ }^{\dagger}$ Author for correspondence. Centre for Transport Studies, Imperial College London, London SW7 2AZ, UK. Email: d.j.graham@imperial.ac.uk, Tel: +44 (0)20 7594 6088, Fax: +44 (0)20 75946107.
} 


\section{Introduction}

In the last decade, a significant number of European Union directives have proposed vertical separation of rail infrastructure management from the operation of rail services and have also promoted open access to new operators (e.g. Council Directive 91/440/EEC, 95/18/EC, 95/19/EC and 2001/13/EC). The aim of these directives has been to encourage internal competition in the European railway market and to address the trends over recent decades that have culminated in a falling market share of European railways and an increasing need for subsidies.

The EU proposals were based on the conviction that competitive pressure, and the competitive strategy of railway firms, would result in a reduction of subsidies and operation costs by eliminating inefficiencies and encouraging revenue maximisation. However, if there has been some consensus in the last decade about the need to stimulate contestable markets, it seems that recent difficulties in implementation and in the actual process of reform have raised the possibility that we may be able to achieve only marginal and temporary contestability (see Bognetti and Fazioli 1999). Issues such as these have motivated research into the economic characteristics of the railway industry, aiming to predict which policy environments and production structures can give rise to increased efficiency and productivity and allow the railway sector to become more competitive.

While there is an extensive literature on rail productivity and efficiency, most studies to date have concentrated on identifying particular sources of productivity. In this paper we estimate the sources of efficiency and productivity within the European railway industry, but also identify their main determinants including firm and country specifics effects and the influence of policy restructuring processes. The analysis makes use of recent developments in econometrics to estimate a cost 
frontier that isolates three main sources of productivity: firm efficiency, returns to scale and technological progress. Using two alternative assumptions regarding the nature of allocative inefficiency the cost frontier analysis allows us to identify the relative contributions of allocative and technical inefficiencies in the cost performance of firms. Following the methodology set out in Bauer (1990a), the growth rate of total factor productivity (TFP) is then calculated allowing us to distinguish movements of the cost function that are due to embodied and disembodied technological progress, changes in efficiency of firms, and movements along the cost function due to economies of scale.

In addition to these substantive contributions the paper also makes methodological innovations through the choice and measurement of the variables used for estimation. In addition to the variables typically used in railway econometric studies, this paper provides a re-evaluation of capital stock and introduces new exogenous variables such as environmental characteristics and quality of service variables, which were developed to improve model specification and to allow for the analysis of firm specific and technological effects.

The paper is organised as follows. Section II describes the stochastic approach to minimum cost function estimation and efficiency analysis. Section III describes the data and the variables used in our model. Econometric results and analyses of cost inefficiencies and the growth of total factor productivity (TFP) are provided in section IV. Conclusions are drawn in the final section. 


\section{The Stochastic Frontier Approach}

The traditional cost function approach assumes that the producer is efficient in reaching the cost minimisation objective. The only error in this model is white noise and possible model misspecification. The stochastic cost function recognises that a cost-minimising firm may not be able to produce a given level of output, with given input prices, at a minimum cost due to the existence of technical and allocative inefficiencies which cause deviations from the cost frontier. Technical inefficiency is the failure to produce the maximum possible output with some given a set of inputs. Allocative inefficiency arises from adopting a wrong, or sub-optimal, choice of input-proportions given input prices. Both types of inefficiencies increase total cost.

The cost frontier is rendered stochastic through the inclusion of white noise error, which could represent exogenous shocks that are outside the control of firms, the use of inappropriate data, measurement error, and sources of model misspecification. White noise may increase or decrease cost and gives rise to the following specification of the cost function:

$$
\ln C=\ln C^{*}+\varepsilon
$$

where $C$ is total or variable cost, $C^{*}$ defines the deterministic cost frontier, and $\varepsilon$ comprises technical $\left(C_{\tau}\right)$ and allocative $\left(C_{a}\right)$ inefficiencies as well as a random term. Thus the cost equation (1) can be rewritten as:

$$
\ln C=\ln C^{*}+\ln C_{\tau}+\ln C_{a}+v
$$

The appearance of $\mathrm{C}_{\tau}$ and $\mathrm{C}_{a}$ in $\log$ linear form allows one to express the actual costs (discarding the white noise component) supported by firms as the product of three impendent elements: the 
minimum or frontier cost $\left(C^{*}\right)$, the cost of allocative inefficiency $\left(C_{a}\right)$ and the cost of technical inefficiency $\left(C_{t}\right)$ (Farrell 1957).

The factor shares can be obtained by differentiating the previous expression of the logarithm cost with respect to the logarithm of each factor price $\left(\mathrm{w}_{\mathrm{i}}\right)$, as follows:

$$
\mathrm{S}_{\mathrm{i}}=\frac{\partial \ln \mathrm{C}}{\partial \ln \mathrm{w}_{\mathrm{i}}}=\frac{\partial \ln \mathrm{C}^{*}}{\partial \ln \mathrm{w}_{\mathrm{i}}}+\frac{\partial \ln \mathrm{C}_{\mathrm{a}}}{\partial \ln \mathrm{w}_{\mathrm{i}}}+\alpha_{\mathrm{i}},
$$

where $\alpha_{i}$ indicates a component error term. Since the cost of technical inefficiency is, by definition, independent of input prices, there is no explicit term of technical inefficiency in cost share equations.

Brown and Walker (1995) argue that in a model consisting of the share equations and the cost function, the share residuals should not contain the additive functionally independent errors, that is, the noise component $\alpha_{\mathrm{i}}$. They found that the use of an additive, homoskedastic error structure, functionally independent of input prices and output levels, for a complete system of equations derived from cost minimisation, either violates the basic restriction from production theory or restricts the form of the underlying technology. Thus, based on this theoretical view, the share equations, as ancillary functions, only reproduce the piece of information contained in the cost function that depends on inputs prices, and, as a consequence, the deviations from each optimal share equation are exclusively due to an over or underutilisation of the input considered (the allocative inefficiency). However, we can also argue that the actual share observations $S_{i}$, by their own empirical nature, incorporate stochastic terms that are clearly independent of the model, and as such potentially include measurement errors that could bias the residual based measure of allocative inefficiency. 


\begin{abstract}
The development of a consistent model of the cost-share system which incorporates the heteroskedastic term in the cost function (resulting from the additive error term of share equations) is an extremely difficult task (see Brown et al 1995). We approach this problem by specifying alternative estimation models corresponding to both hypotheses: one with, and one without, the stochastic noise term $\alpha_{i}$ in the cost share equations.
\end{abstract}

Thus, generically the share equation presented above can be written in the following form:

$$
S_{i}=\frac{\partial \ln C}{\partial \ln w_{i}}=\frac{\partial \ln C^{*}}{\partial \ln w_{i}}+u_{i}
$$

where $u_{\mathrm{i}}$ represents the random deviation term to optimal input shares, and which, depending on the hypothesis considered, includes or does not include the stochastic noise term.

The cost function can be estimated after specifying a functional form for $\ln C^{*}$ and making some distributional assumptions regarding $\ln C_{\tau}$ and $\operatorname{lnC}_{a}$. However, since the cost of allocative inefficiency is not independent of input prices, then in order to obtain consistent parameters estimates it is necessary to separate the costs of technical and allocative inefficiencies through some additional assumptions. Specifically, we need to link allocative inefficiency as indirectly measured by the cost share equation residuals $u_{\mathrm{i}}$, and the cost of allocative inefficiency $\left(\ln C_{a}\right)$ in the cost function.

We model the deterministic component of the cost function using the translog form: 


$$
\begin{aligned}
& \ln C^{*}=\alpha_{0}+\gamma^{\prime} \ln y+\alpha^{\prime} \ln w+\eta^{\prime} \ln q+\ln y \Omega \ln w+\ln w \Phi \ln q+\ln y \Psi \ln q \\
& +\frac{1}{2} \ln y \Pi \ln y+\frac{1}{2} \ln w \mathrm{~B} \ln w+\frac{1}{2} \ln q \Delta \ln q
\end{aligned}
$$

where $y$ is a vector of outputs, $w$ is a vector of input prices, and $q$ is a vector of exogenous variables which include a time trend, firm specific technical attributes, the characteristics of the general operational environment characteristics, and the nature of quasi-fixed factors.

The dependent variable $\ln C^{*}$ is defined as the firms' variable cost ${ }^{1}$. In addition to the symmetry restrictions on the parameter matrices $\mathrm{B}, \Pi$ and $\Delta$, we impose the following restrictions: $\alpha^{\prime} l=1$, $\mathrm{B}^{\prime} l=0, \Omega^{\prime} l=0, \Phi^{\prime} l=0$, where $l$ is a vector of ones. These restrictions on parameter vectors make the cost function in (5) linear homogeneous in input prices. By applying Shephard's lemma to (5) we derive the deterministic part of cost shares equations

$$
S_{i}^{*}=\alpha_{i}+\sum_{j=1}^{n} \beta_{i j} \ln w_{j}+\sum_{k=1}^{m} \omega_{i k} \ln y_{k}+\sum_{l=1}^{r} \varphi_{i l} \ln q_{l}, \mathrm{i}=1, \ldots, \mathrm{n} .
$$

\footnotetext{
Estimation of the model given in (2) and (4) requires some specification of distributional assumptions for $\ln \mathrm{C}_{\mathrm{\tau}}, \ln \mathrm{C}_{a}$ and $u_{\mathrm{i} .}$ Regarding distribution of the composite error vector $\varepsilon$ [equation (1)] we assume that:
}

(i) $\quad u_{\mathrm{i}}$ is distributed independently and identically through firms as multivariate normal with zero mean and constant covariance $\mathrm{S}$,

(ii) $\quad \ln C_{\tau}$ is independently and identically distributed following a Half-Normal distribution $N\left(0, \sigma_{\tau}\right)$ - corresponding to the absolute value of a normally distributed variable with mean zero and standard deviation $\sigma_{\tau}$, 
(iii) the random noise, $\mathrm{n}$, is independently and identically distributed according to a normal distribution - $N\left(0, \sigma_{v}\right)$,

(iv) the error components $\ln C_{\tau}, u_{\mathrm{i}}$, and $v$ are assumed to be mutually independent over years and across firms.

With these distributional assumptions, the concentrated log-likelihood function $\mathrm{L}$ for a sample of $\mathrm{F}$ firms observed over T years, following the same derivation used by Kumbhakar (1991) and Parisio (1999), is given by :

$$
L_{c}=\text { const } .-\frac{1}{2} F \cdot T \cdot \ln |\Sigma|-F \cdot T \cdot \ln \sigma-\frac{\sum_{f=1}^{F} \sum_{t=1}^{T} \zeta_{f t}^{2}}{2 \sigma^{2}}+\sum_{f=1}^{F} \sum_{t=1}^{T} \ln \left[1-\Phi\left(-\frac{\lambda \zeta_{f t}}{\sigma}\right)\right] \text {, }
$$

where, $f$ and $t$ index firm and time $(f=1, \ldots \mathrm{F}, t=1, \ldots \mathrm{T}), \zeta_{f t}=\left(\varepsilon_{f t}-\ln C_{a_{f t}}\right), \sigma^{2}=\left(\sigma_{v}^{2}+\sigma_{\tau}^{2}\right)$, $\lambda=\sigma_{\tau} / \sigma_{v}, \Phi($.$) is the standard normal distribution function and the (i,j) element of \Sigma, \sigma_{i j}$, is:

$$
\sigma_{i j}=\frac{1}{F T} \sum_{f} \sum_{t} u_{i f t} u_{j f t}=\frac{1}{F T} \sum_{f} \sum_{t}\left(S_{i f t}-S_{i f t}^{*}\right)\left(S_{j f t}-S_{j f t}^{*}\right) .
$$

Given the relationship between random terms and allocative inefficiency, the maximum likelihood estimates of the parameters in equations (5) and (6) can be obtained by maximising the loglikelihood function $\mathrm{L}_{\mathrm{c}}$, where the elements of $\Sigma$ are given by relation (8).

The relationship between the allocative inefficiency component in the cost equation and the deviations to optimal cost shares in equation (4) can be explicitly modelled for the translog system by means of alternative specifications taking into account the differentiability condition and the fact that $\operatorname{lnC}_{a}\left(u_{i}\right) / 0$. The specification choice depends on the nature of the assumptions made about the structure of the error terms $u_{\mathrm{i}}$ and the level of flexibility imposed on the system. Specifically, it 
depends on the possible pre-violation of the regularity and of the integrability conditions which we are willing to tolerate in order to benefit from the empirical advantages that arise from use of a more flexible estimation technique.

In this context, we propose two alternative models establishing the relationship through an approximating function imposing all the structure we know a priori. These models are based on the Schmidt and Sickles (1984) proposed specification where $\operatorname{lnC}_{a}$ is formulated as :

$$
\ln C_{a}=U^{\prime} K U
$$

where $\mathrm{K}$ is a pre-specified positive matrix and $\mathrm{U}=\left(u_{1}, u_{2}, \ldots u_{\mathrm{n}-1}\right)^{\prime}$. In this specification, the cost of allocative inefficiency is a weighted average of the squared cost share errors. Thus, the cost is increased as the magnitude of the absolute cost share errors increases, always being positive if at least one $u_{\mathrm{i}}$ is different from zero. In this study the pre-specific matrix $\mathrm{K}$ requires a simplification relative to Schmidt's specification to obtain a more tractable maximum-likelihood procedure. Following the suggestion proposed by Bauer (1990b), we set the matrix K to be a symmetric matrix where its elements are separate parameters to be estimated. Under this specification, no regularity conditions are imposed a priori and, moreover, the integrability condition associated with the application of Shepard's Lemma to the allocative cost term and to equations (3) or (4) is not satisfied.

The two models used to estimate the allocative inefficiency are as follows.

i. Model I gives a solution with the cost share error term exclusively indexed to allocative errors. In this model the allocative inefficiency is calculated from the relation (4), where the matrix $\mathrm{K}$ is assumed to be a diagonal matrix and the parameters of this diagonal matrix are to be jointly estimated with the remaining parameters of the cost function. In this approximation method, the allocative inefficiency of the cost term appears in the cost 
function as a linear function of the error terms $u_{\mathrm{i}}$ of the cost share equation and can be expressed as:

$$
\ln C_{a}=\sum_{i=1}^{n-1} C_{i}\left(u_{i}^{2}\right)
$$

where $C_{\mathrm{i}}$ are the parameters to be estimated and $u_{\mathrm{i}}$ is the error term of the $i^{\text {th }}$ input cost share equation.

ii. Model II gives a solution with the cost share term including allocative and noise errors. The second approximate solution model has been formulated on the basis that the error term in the cost share equations is composed of a functionally dependent error term, the $\alpha_{i}^{a}$, and the noise term $\alpha_{i}$. The allocative error term, $\alpha_{i}^{a}$, is considered to be a linearly dependent function of the input prices, a time trend variable and a firm specific effect variable. Thus, as in the first model, assuming the cost of allocative inefficiency given by the relation (9), where the matrix $\mathrm{K}$ was pre-specified as a symmetric matrix, the term $\ln C_{a}$ would be given by the following formulation:

$$
\ln C_{a}=\sum_{i=1}^{n-1} \sum_{j=1}^{n-1} k_{i j} \alpha_{i}^{a} \alpha_{j}^{a}
$$

where $k_{\mathrm{ij}}$ are the elements of matrix $K$ to be estimated with $k_{\mathrm{ij}}=k_{\mathrm{ji}} ; \alpha_{i}^{a}$ is given by:

$$
\alpha_{i}^{a}\left(w_{j}, t, D_{f}\right)=\sum_{j=1}^{n} \omega_{i j} w_{j}+\sum_{f=1}^{F} \varpi_{i f} D_{f}(1+\log t)^{-1}+\sum_{f=1}^{F} \delta_{i f} D_{f}
$$

where the parameters $\omega_{i j}, \varpi_{i f}$ and $\delta_{i f}$ are parameters to be estimated. Note that the time trend formulation $[1+\log (t)]^{\wedge(-1)}$ has been used instead of a linear one. The reason for this is that since efficiency gains are not a continuous source of productivity, gains from correcting allocative inefficiency (implying decreasing inefficiency over time) become increasingly more difficult after previous years, and consequently, a constant marginal efficiency gain 
over time would not characterise this effect. The $D_{f}$ variables are firm specific dummy variables.

Finally in this section, we turn to the calculation of technical efficiency. Since the cost of technical inefficiency is independent of input prices in a cost minimisation framework, the random term characterising the cost of technical inefficiency $\left(\operatorname{lnC}_{\tau}\right)$ gives the maximum rate at which the use of all the input can be reduced without reducing output. Thus $\operatorname{lnC}_{\tau}(\geq 0)$ measures input-saving technical inefficiency. Ideally, we would estimate $\operatorname{lnC}_{\tau}$ for each firm in the sample to compare them on the basis of their productivity inefficiency. Unfortunately, this is not directly estimable since, with parameters estimates in hand, we are only able to compute a direct estimate of $\left(\ln C_{\tau}+v\right)$. Jowdrow et al. (1982), however, have derived a useful approximation that is now the standard measure in these settings, and which will be applied here ${ }^{2}$. Using the maximum likelihood estimates of the structural parameters in the model and the assumed half-normal distribution for the random variable $\left(\tau=\ln C_{\tau}\right.$ ), the proposed approximation for technical inefficiency measure is given by:

$$
E[\tau \mid \varsigma]=\frac{\sigma \lambda}{1+\lambda^{2}}\left[\frac{\phi(z)}{1-\Phi(z)}-z\right], \text { with } z=\frac{\varsigma \lambda}{\sigma}
$$

where $\zeta=\left(\varepsilon-\ln C_{a}\right), \phi($.$) and \Phi($.$) are respectively the standard normal density and distribution$ function, $\sigma^{2}=\left(\sigma_{v}^{2}+\sigma_{\tau}^{2}\right)$ and $\lambda=\sigma_{\tau} / \sigma_{v}$ as defined above. 


\section{Data.}

The cost system that we have derived is composed in its deterministic part by three different types of exogenous variables ${ }^{3}$ : output, input prices and the operational attribute variables. The data we have available to represent this cost system are for twenty seven firms of European countries over the period 1972 to 1999 (Table 1) (see appendix 1) ${ }^{4}$. These data were obtained from the International Union of Railways (UIC) and additionally supplemented with information from Jane's (1995), ECIS (1996), Uden (2001) and firm annual reports.

Table 1 -Data for cost system estimation.

\begin{tabular}{llll}
\hline \multicolumn{1}{c}{ FIRMS } & DATA PERIOD & FIRMS & DATA PERIOD \\
\hline CFF (SBB/CFF/FFS) & $1972-1999$ & NSB & $1972-1993$ \\
CFL & $1972-1999$ & BR & $1972-1994$ \\
CH & $1972-1999$ & DSB & $1972-1996$ \\
CIE & $1972-1999$ & CD (CSD) & $1990-1999$ \\
CP (CP / REFER) & $1972-1999$ & PKP & $1991-1999$ \\
DB (DBAG) & $1972-1999$ & MÁV & $1991-1999$ \\
FS (FS SpA) & $1972-1999$ & BC & $1992-1999$ \\
NS (NS B.V. / N.V.) & $1972-1999$ & BDZ & $1992-1999$ \\
ÖBB & $1972-1999$ & CFR & $1992-1999$ \\
RENFE & $1972-1999$ & EVR & $1992-1999$ \\
SJ (SJ/BV) & $1972-1999$ & SZ & $1992-1999$ \\
SNCB (SNCB/NMBS) & $1972-1999$ & ZSR & $1993-1999$ \\
SNCF (SNCF / RFF) & $1972-1999$ & & \\
TCDD & $1972-1999$ & & \\
VR (VR / RHK) & $1972-1999$ & & \\
\hline
\end{tabular}

As mentioned previously, the dependent variable is the short run variable cost. The infrastructure capital stock variable enters the function as an exogenous and fixed quantity (in a short-run perspective) and includes the "land and fixed installations" as defined in reported UIC data. The cost, prices and productive capital stock of infrastructure were estimated following the criteria 
defined in OECD-Statistics (March-2001) and OECD-Statistics (2001a), where the cost of capital was estimated as a user cost, defined as the sum of depreciation ${ }^{5}$ and interest costs. The productive capital stock $\left(K_{\mathrm{t}}\right)$ is the stock of a particular asset, after assets of different ages have been converted into standard efficiency units, thus adjusting the older assets in the stock to account for their reduced efficiency in producing capital services. Productive capital stock is a proxy for the quantity of capital services produced by the asset, not directly observable, and thus assumed to be in proportion to the stock of assets after each vintage has been converted into standard efficiency units.

Thus, productive capital stock was given by the following expression:

$$
K_{t}=\sum_{\tau=0}^{T} h_{\tau} \cdot F_{\tau} \frac{I N_{t-\tau}}{q_{t-\tau, 0}}
$$

where, IN is the nominal investment expenditure ${ }^{6}$ on the asset at time $t, q_{t, 0}$ is the price index deflator for the asset of age zero (a new asset) in year $t, F_{t}$ is a retirement function that spells out the share of asset of age $t$ that is still in service, $\mathrm{T}$ is the maximum service life of asset, and $h_{\mathrm{t}}$ is an age-efficiency profile, tracing the loss in productive efficiency as an asset ages ${ }^{7}$.

The variable input prices used in this study are labour (LB), services rendered by third parts (EM), which include energy and materials consumption, and equipment capital stock (EQ). The use of aggregated inputs was necessary due to the lack of available homogeneous disaggregate input data. Constant cost and price data for the sample have been calculated using the World Bank country price deflators and the OECD country Purchasing Power Parities (PPPs). The variable definitions are as follows. 
i. The labour input price variable is measured, as is common in the literature, by the ratio of staff charges to annual mean staff. Staff charges are the sum of expenses from salaries plus pensions and various social charges.

ii. Ideally, materials and energy would be constructed as two distinct variables. However, for this study, disaggregate data on both inputs are not available and instead we use the ratio of materials and energy costs over the total train-kilometres supplied by each company. This approximation of the prices of material and energy inputs is similar to that proposed by Preston (1994) and Sánchez and Villarroya (2000). The use of an aggregate measure is less problematic due to the empirical proportionality that is typically found between material and energy consumption and train-kilometres supplied.

iii. Capital stock equipment includes the transport stock and other equipment fixed assets. The cost, prices and productive capital $\operatorname{stock}^{8}$ of "capital stock equipment" were estimated following the same procedure used to derive the capital stock infrastructure. The rate of depreciation $\left(d_{t}\right)$ was calculated as the change of the net capital stock at constant prices ${ }^{9}$ and the real cost of financial capital $\left(r_{t}\right)$ was measured as a return on government bonds given by the long term real interest rates for government bonds reported in IMF (2001), World Bank (2002) and OECD (2002).

For the vector of aggregated output representing the production of the passenger (PKM) and freight services (TKM), either passenger-kilometres and ton-kilometres or passenger train-kilometres and freight train-kilometres could be used. Here we use both alternative output vectors to provide a comparison of productive efficiency results. Using passenger-kilometres and ton-kilometres as output $\left(\mathrm{Y}_{\mathrm{I}}\right)$ the inefficiency results include, apart from managerial inefficiency, the inefficiency that arises through effects such as subsidies, governmental and/or regulatory control. The other model 
group $\left(\mathrm{Y}_{\mathrm{II}}\right)$ uses passenger train-kilometres and freight train-kilometres and consequently the empirical inefficiency results can be interpreted exclusively as firm managerial efficiency effects.

The operational attribute variables $(q)$ in the system of equations (5) and (6) represent a variety of exogenous variables related to the geographical environment of the firm and the characteristics of firm technology.

The variables we use to characterise the physical environment where the firms operate are: the length of network (NET), the population density of the country (DENS) (World Bank 2002); the number of metropolitan cities potentially served by the firm network with more than one million inhabitants (AGM) (Brinkhoff 2002); the average number of days per year that have snow in each country (SNOW) (ERSI 2002); the weighted average of the gradient level that characterises the differences in terrain in each country (SURVEY) (Maps.Com 1999).

The variables that characterise the technological differences between firms and over time are:

i. a linear time trend that captures technical progress from the shift in the cost function over time (TIME);

ii. a set of dummy variables capturing the effects of introduction and use of high speed lines (HS220, TT, DHS, DTT) ${ }^{10}$ and

iii. a proxy index number variable quantifying the operational quality level provided by firms. This variable (QVMAX) mainly takes into account factors related to the potential "network" velocity and works out as a complement velocity effect not captured by high speed dummy variables. Specifically, we collect information on the potential maximum 
8

velocity of tractive stock, the frequency of service, the percentage of electrified network, and the percentage of double track. To overcome potential problems of multicollinearity, the QVMAX variable was calculated as the first principal component of these four original variables after they have been standardised to have unit variance. Use of the method of principal components means that the service quality variable is an index without units of measurement.

Table 2 gives a summary of some basic characteristics of the companies that comprise our sample of European railways. 
Table 2 - Basic characteristics of the European railway systems

\section{Results}

\begin{tabular}{|c|c|c|c|c|c|c|c|c|c|c|c|c|c|}
\hline \multirow[b]{2}{*}{ Firms } & \multicolumn{8}{|c|}{$\begin{array}{l}\text { Basic Network Characteristics } \\
\text { (Mean value during sample period) }\end{array}$} & \multicolumn{5}{|c|}{$\begin{array}{c}\text { Basic Cost Characteristics } \\
\text { (Mean value during sample period) }\end{array}$} \\
\hline & $\begin{array}{l}\text { NET } \\
(\mathrm{km})\end{array}$ & APTD & AFTD & $\begin{array}{c}\text { PTRD F } \\
\left({ }^{*} 1000\right)\end{array}$ & $\begin{array}{l}\text { FTRD } \\
\left({ }^{*} 1000\right)\end{array}$ & $\begin{array}{l}\text { ALT } \\
(\mathrm{km})\end{array}$ & $\begin{array}{cc}\text { ALH } & 0 \\
(\mathrm{~km}) & (\end{array}$ & $\begin{array}{r}\text { Qvmax } \\
\text { (Index) }\end{array}$ & $\begin{array}{l}\text { VC_TK } \\
(\text { US } \$ / k m)\end{array}$ & $\begin{array}{r}\text { SHLB } \\
(\%)\end{array}$ & $\begin{array}{r}\text { SHEM } \\
(\%)\end{array}$ & $\begin{array}{r}\text { SHEQ } \\
(\%)\end{array}$ & $\begin{array}{l}\text { TK_S } \\
(\mathrm{km} / \mathrm{empl} .)\end{array}$ \\
\hline BR & 17303 & 20.0 & 4.4 & 1802.4 & 1036.7 & 42.7 & 121.6 & 4.2 & 19.3 & 56.73 & 36.74 & 6.52 & 2.52 \\
\hline$C D$ * & 9417 & 10.6 & 5.0 & 818.6 & 2181.2 & 37.6 & 203.3 & 1.5 & 23.9 & 37.73 & 44.53 & 17.75 & 1.51 \\
\hline CFF & 2951 & 26.9 & 9.4 & 3409.8 & 2456.9 & 42.1 & 159.3 & 5.1 & 24.4 & 61.09 & 23.32 & 15.59 & 3.00 \\
\hline CFL & 272 & 13.9 & 5.3 & 900.3 & 2366.0 & 22.2 & 37.8 & 4.2 & 51.5 & 78.14 & 14.73 & 7.14 & 1.59 \\
\hline $\mathrm{CH}$ & 2467 & 5.6 & 1.1 & 686.3 & 259.1 & 144.0 & 195.8 & 0.4 & 30.9 & 59.70 & 20.77 & 19.53 & 1.40 \\
\hline CIE & 1984 & 4.5 & 2.1 & 552.1 & 293.5 & 55.2 & 176.3 & 1.5 & 45.8 & 57.19 & 32.57 & 10.24 & 2.13 \\
\hline $\mathrm{CP}$ & 3393 & 8.4 & 2.0 & 1513.3 & 407.4 & 26.7 & 237.5 & 1.1 & 22.2 & 58.81 & 25.22 & 15.97 & 1.70 \\
\hline CP/REFER * & 2821 & 13.8 & 2.8 & 1594.6 & 889.9 & 26.0 & 238.5 & 2.2 & 16.8 & 44.44 & 41.24 & 14.32 & 3.61 \\
\hline$D B$ * & 27988 & 14.4 & 7.0 & 1456.7 & 2135.2 & 39.1 & 206.7 & 3.0 & 31.3 & 72.66 & 21.15 & 6.19 & 2.09 \\
\hline DB AG & 39674 & 16.8 & 5.2 & 1571.4 & 1790.4 & 43.9 & 241.7 & 3.6 & 15.5 & 49.40 & 46.66 & 3.94 & 3.38 \\
\hline DSB & 2223 & 18.9 & 3.5 & 1832.0 & 809.7 & 33.9 & 234.8 & 2.6 & 22.3 & 48.31 & 31.31 & 20.38 & 3.00 \\
\hline EVR * & 1001 & 4.7 & 3.5 & 454.9 & 4468.8 & 45.1 & 158.3 & 0.4 & 24.0 & 37.74 & 56.53 & 5.73 & 1.16 \\
\hline FS & 16114 & 14.5 & 3.8 & 2601.9 & 1211.1 & 102.5 & 325.1 & 3.2 & 39.1 & 60.74 & 27.89 & 11.38 & 1.66 \\
\hline MAV * & 7665 & 9.4 & 2.6 & 856.6 & 1072.8 & 51.1 & 173.2 & 1.4 & 21.2 & 41.71 & 34.58 & 23.72 & 1.44 \\
\hline NS & 2832 & 35.6 & 4.5 & 3624.8 & 1078.0 & 44.9 & 157.1 & 5.6 & 14.8 & 54.21 & 29.26 & 16.53 & 4.19 \\
\hline NS (B.V./N.V.) * & 2807 & 41.0 & 2.3 & 5220.7 & 1279.9 & 46.0 & 151.2 & 6.9 & 24.9 & 32.66 & 57.58 & 9.76 & 4.65 \\
\hline NSB & 4187 & 5.5 & 2.5 & 506.1 & 642.0 & 61.0 & 110.2 & 1.0 & 19.6 & 63.83 & 23.90 & 12.27 & 2.49 \\
\hline OBB & 5740 & 12.4 & 6.6 & 1352.8 & 2041.8 & 44.9 & 203.0 & 2.8 & 29.7 & 51.65 & 27.09 & 21.26 & 1.71 \\
\hline PKP * & 24131 & 7.5 & 4.2 & 1014.3 & 2620.5 & 59.2 & 301.3 & 2.3 & 29.6 & 34.20 & 30.25 & 35.55 & 1.22 \\
\hline RENFE & 12997 & 8.3 & 3.4 & 1212.7 & 898.2 & 68.0 & 363.8 & 2.2 & 24.0 & 51.12 & 30.20 & 18.69 & 2.85 \\
\hline SJ & 11389 & 5.3 & 3.6 & 515.3 & 1407.7 & 83.7 & 305.7 & 1.6 & 17.1 & 65.03 & 22.12 & 12.86 & 3.07 \\
\hline $\mathrm{SJ} / \mathrm{BV}$ & 10230 & 6.0 & 3.6 & 603.9 & 1733.6 & 67.2 & 394.9 & 2.5 & 18.7 & 40.68 & 47.99 & 11.33 & 4.84 \\
\hline SNCB & 3711 & 19.1 & 5.6 & 1865.6 & 2250.4 & 43.9 & 114.9 & 5.0 & 37.9 & 60.96 & 25.13 & 13.91 & 1.97 \\
\hline SNCF & 33958 & 8.8 & 5.4 & 1659.6 & 1737.3 & 77.2 & 336.7 & 3.2 & 26.5 & 55.58 & 27.54 & 16.88 & 2.19 \\
\hline SNCF/RFF * & 31715 & 11.1 & 4.9 & 2018.8 & 1934.5 & 78.1 & 384.8 & 4.1 & 29.3 & 43.52 & 50.51 & 5.97 & 3.03 \\
\hline $\mathrm{SZ}$ * & 1201 & 9.3 & 6.0 & 499.0 & 2207.4 & 45.1 & 196.4 & 2.1 & 25.7 & 45.59 & 36.61 & 17.80 & 1.86 \\
\hline TCDD & 8297 & 2.8 & 2.2 & 715.9 & 877.9 & 49.0 & 509.2 & 0.6 & 35.7 & 54.56 & 33.10 & 12.33 & 0.93 \\
\hline VR & 5961 & 4.2 & 3.1 & 517.7 & 1285.1 & 78.4 & 264.8 & 1.2 & 18.4 & 69.77 & 21.54 & 8.56 & 1.98 \\
\hline VR/RHK * & 5857 & 4.5 & 2.9 & 572.9 & 1634.9 & 66.7 & 241.1 & 1.7 & 16.2 & 45.88 & 47.31 & 6.81 & 4.43 \\
\hline ZSR * & 3666 & 10.3 & 6.3 & 1023.2 & 3345.5 & 46.5 & 210.6 & 2.0 & 24.1 & 41.44 & 46.96 & 11.60 & 1.24 \\
\hline \multicolumn{14}{|c|}{ mean firm values from a sample with less then ten years observations } \\
\hline NET: & \multicolumn{8}{|c|}{ Network length (kms) } & VC_TK & \multicolumn{4}{|c|}{ Variable costs per train kilometres } \\
\hline APTD: & \multirow{2}{*}{\multicolumn{8}{|c|}{$\begin{array}{l}\text { Average passenger train density (passenger train-kms per route } \mathrm{kms} \text { ) } \\
\text { Average freight train density (freight train-kms per route kms) }\end{array}$}} & SHLB: & \multicolumn{4}{|c|}{ Average labour cost shares (\%) } \\
\hline AFTD: & & & & & & & & & SHEM: & \multirow{2}{*}{\multicolumn{4}{|c|}{$\begin{array}{l}\text { Average energy\&materials cost sha } \\
\text { Average equipment cost share (\%) }\end{array}$}} \\
\hline PTRD: & \multicolumn{8}{|c|}{ Passenger traffic density (passenger-kms per route $\mathrm{kms}$ ) } & SHEQ: & & & & \\
\hline FTRD: & \multirow{2}{*}{\multicolumn{8}{|c|}{ Freight traffic density (tonne-kms per route $\mathrm{kms}$ ) }} & TK_S: & \multirow{2}{*}{\multicolumn{4}{|c|}{ Train-kilometres per number of employees }} \\
\hline ALT: & & & & & & & & & & & & & \\
\hline ALH: & \multicolumn{8}{|c|}{$\begin{array}{l}\text { Average passenger trip length (passenger-kms per pa } \\
\text { Average freight length of haul (tonne-kms per tonne) }\end{array}$} & & & & & \\
\hline Qvmax: & \multicolumn{8}{|c|}{ Quality of service index } & & & & & \\
\hline
\end{tabular}

The results section is organised as follows. First we present parameters estimates from cost function estimation for our two model using two separate output vectors. Next, we use the estimates to construct measures of technical and allocative inefficiencies for the railways. We then present the results of some further regressions to identify the sources of cost inefficiencies. Finally, we present a decomposition of TFP growth. 


\section{Parameter estimates from the cost functions}

Initial estimation of the general cost function (5) revealed evidence of severe multicollinearity. Following Klein (1962), the correlation matrix of all terms in the deterministic cost function was calculated, and the variable terms with high levels of correlation were excluded. The exclusion of these variables did not imply restrictions either in the flexibility of the function (still allowing variable elasticity of substitution) nor in the theoretical properties of the cost function.

Preliminary estimation results using the standard infrastructure productive capital stock, KQ, showed frequent violations of curvature conditions, with positive estimates for the parameter associated with the second order quadratic KQ term, directly contradicting cost theory. To solve this problem, quasi-fixed capital stock, KQ, was replaced by a measure of the service flow from capital as the argument in the variable cost function, as suggested by Oum and Zhang (1991). To compute infrastructure capital stock service flow, $\mathrm{KQ}^{*}$, we need to multiply the infrastructure productive capital stock (KQ) by its utilisation rate. Since the utilisation rate of infrastructure productive capital stock is not directly observable, some proxy must be constructed. Therefore the utilisation rate (CUK) was defined as the ratio of total train-kilometres run (per year) per kilometre of line to the maximum value of total train-kilometres run (per year) per kilometre of line observed in the present sample.

The restriction of linear homogeneity in the input prices was imposed by normalising variable costs and the other prices by the price of materials \& energy input (PEM). To avoid singularity in the disturbance variance-covariance matrix, the materials \& energy input cost share equation was 
dropped from estimation. It is well known that the maximum likelihood estimates are invariant to the choice of the share equation dropped.

Thus, the final system for estimation comprising a variable cost functions and input cost shares is:

$$
\begin{aligned}
& \ln \left(\frac{V C}{P E M}\right)=\alpha_{0}+\alpha_{L B} \ln \left(\frac{P L B}{P E M}\right)+\alpha_{E Q} \ln \left(\frac{P E Q}{P E M}\right)+\gamma_{P K M} \ln (P K M)+\gamma_{T K M} \ln (T K M)+\eta_{Q V \max } \ln (Q v \max ) \\
& +\eta_{\text {Net }} \ln (N E T)+\eta_{K Q} \ln \left(K Q^{*}\right)+\eta_{\text {Time }}(T I M E)+\omega_{L B P K M} \ln \left(\frac{P L B}{P E M}\right) \cdot \ln (P K M)+\omega_{E Q P K M} \ln \left(\frac{P E Q}{P E M}\right) \cdot \ln (P K M) \\
& +\omega_{E Q T K M} \ln \left(\frac{P E Q}{P E M}\right) \cdot \ln (T K M)+\phi_{L B T i m e} \ln \left(\frac{P L B}{P E M}\right) \cdot(T I M E)+\phi_{L B Q V \max } \ln \left(\frac{P L B}{P E M}\right) \cdot \ln (Q v \max ) \\
& +\phi_{E Q K Q} \ln \left(\frac{P E Q}{P E M}\right) \cdot \ln \left(K Q^{*}\right)+\psi_{\text {PKMKQ }} \ln (P K M) \cdot \ln \left(K Q^{*}\right)+\psi_{\text {YKMKQ }} \cdot \ln (T K M) \cdot \ln \left(K Q^{*}\right) \\
& +\frac{1}{2} \beta_{L B L B}\left[\ln \left(\frac{P L B}{P E M}\right)\right]^{2}+\beta_{L B E Q} \ln \left(\frac{P L B}{P E M}\right) \cdot \ln \left(\frac{P E Q}{P E M}\right)+\frac{1}{2} \lambda_{\text {QVQV }}[\ln (Q v \max )]^{2}+\lambda_{\text {QVTime }} \ln (Q v \max ) .(T I M E) \\
& +\lambda_{K Q K Q}\left[\ln \left(\frac{K Q^{*}}{N E T}\right)\right]^{2}+\kappa_{\text {Snow }} \ln (S N O W)+\kappa_{\text {Survey }} \ln (S U R V E Y)+\kappa_{\text {DENS }} \ln (D E N S)+\kappa_{\text {AGM }} \ln (A G M) \\
& +\delta_{H S 220}(H S 220)+\delta_{T T}(T T)+\delta_{D H S}(D H S)+\delta_{D T T}(D T T)+\tau+v
\end{aligned}
$$

$$
\begin{aligned}
S H L B= & \alpha_{L B}+\beta_{L B L B} \cdot \ln \left(\frac{P L B}{P E M}\right)+\beta_{L B E Q} \cdot \ln \left(\frac{P E Q}{P E M}\right)+\omega_{L B P K M} \cdot \ln (P K M) \\
& +\phi_{L B T i m e}(T I M E)+\phi_{L B Q V \max } \cdot \ln (Q v \max )+u_{L B}
\end{aligned}
$$

$$
\begin{aligned}
S H E Q= & \alpha_{E Q}+\beta_{L B E Q} \cdot \ln \left(\frac{P L B}{P E M}\right)+\omega_{E Q P K M} \cdot \ln (P K M)+\omega_{E Q T K M} \cdot \ln (T K M) \\
& +\phi_{E Q K Q} \cdot \ln \left(K Q^{*}\right)+u_{E Q}
\end{aligned}
$$

where SHLB and SHEQ are the input cost shares for labour and transport equipment capital stock. 
The two model specifications (model I and model II) were applied to the sample data set, and they were run for the two alternative output specifications $\mathrm{Y}_{\mathrm{I}}$ and $\mathrm{Y}_{\mathrm{II}}$. Table 3 displays the parameter estimates of the cost frontier functions. Overall, the parameter values indicate that the estimated cost function performs fairly well in both models. For instance, we can see that the values of the log-likelihood function are high, the majority of parameter estimates have the intuitively expected signs, and most importantly, the regularity conditions requiring global concavity of the cost function are confirmed by the estimation of the Hicks-Allen own partial elasticities of substitution, which are all negative at the mean sample and practically negative at all points. To ensure that the cost functions are monotonically increasing in prices, the fitted shares were checked and confirmed to be all positive for both models. Additionally, they are found to be increasing in $\mathrm{Y}$ and therefore all the theoretical properties of the cost function are satisfied. 


\section{Table 3 - Cost function parameters estimates}

\begin{tabular}{|c|c|c|c|c|c|c|c|c|c|c|c|}
\hline \multirow[b]{3}{*}{ Parameters } & \multicolumn{5}{|l|}{ Model I } & \multicolumn{6}{|l|}{ Model II } \\
\hline & \multicolumn{2}{|c|}{ Output Set $Y_{1}$} & \multicolumn{3}{|c|}{ Output Set $Y_{\|}$} & \multicolumn{3}{|l|}{ Output Set $Y_{1}$} & \multicolumn{3}{|c|}{ Output Set $Y_{\|}$} \\
\hline & Coeff. & Std.Err. & Coeff. & Std.Err. & & Coeff. & Std.Err. & & Coeff. & Std.Err. & \\
\hline$\alpha_{0}$ & -4.63169 & $0.5898^{* \star *}$ & 3.19616 & 0.401728 * & 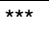 & -4.844440 & 0.525708 * & 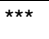 & 5.239590 & 0.363599 & *** \\
\hline$\alpha_{\text {LB }}$ & 1.65855 & $0.1278^{\star \star \star}$ & 0.82500 & 0.0937162 * & $\star \star \star$ & 1.702300 & 0.119958 * & $\star \star \star$ & 0.773276 & 0.080305 & $\star \star \star$ \\
\hline$\alpha_{\mathrm{EQ}}$ & -0.560832 & $0.0361^{* \star *}$ & -0.378212 & 0.0368914 * & 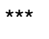 & -0.540094 & 0.034551 * & 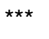 & -0.214595 & 0.030771 & *** \\
\hline$\eta_{\mathrm{KQ}}$ & 0.675204 & 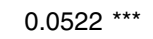 & 0.28364 & 0.0527012 * & $\star \star \star$ & 0.683179 & 0.045825 * & $\star \star \star$ & 0.180675 & 0.046927 & $\star \star \star$ \\
\hline$\eta_{\text {QVmax }}$ & 0.117955 & $0.0291^{* \star *}$ & 0.0580194 & 0.0362897 & & 0.110436 & 0.027362 * & $\star \star \star *$ & 0.044138 & 0.035539 & \\
\hline$\gamma_{\text {PKM }}$ & -0.212865 & $0.0994^{* \star}$ & -0.37727 & 0.153247 * & ** & -0.197686 & 0.093003 * & ** & -0.517170 & 0.163849 & 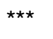 \\
\hline$\gamma_{\text {TKM }}$ & 0.786782 & 0.0902 *** & 0.956365 & 0.15451 * & $\star \star \star$ & 0.812507 & 0.086550 * & $\star \star \star$ & 1.051800 & 0.153613 & $\star \star \star \star ~$ \\
\hline$\delta_{\mathrm{HS} 220}$ & -0.036211 & $0.0094^{\star \star \star}$ & -0.021871 & 0.0096724 * & & -0.044664 & 0.009298 * & $\star \star \star$ & -0.007025 & 0.010554 & \\
\hline$\delta_{\pi}$ & 0.033768 & $0.0113^{\star \star \star}$ & 0.0132182 & 0.0093127 & & 0.023206 & 0.010346 * & $\star \star$ & 0.014572 & 0.009394 & \\
\hline$\delta_{\text {DHS }}$ & 0.038389 & 0.0341 & 0.0007219 & 0.0379293 & & 0.053490 & 0.034433 & & -0.026912 & 0.039429 & \\
\hline$\delta_{\text {DTT }}$ & -0.229561 & $0.0333^{\star \star \star}$ & -0.144623 & 0.0278596 * & 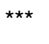 & -0.194635 & 0.026556 * & $\star \star \star *$ & -0.113963 & 0.023740 & $\star \star \star *$ \\
\hline$\kappa_{\text {Snow }}$ & 0.041655 & $0.0057^{\star \star \star *}$ & 0.0079092 & 0.0058846 & & 0.056098 & 0.004609 * & $\star \star \star$ & 0.007905 & 0.004894 & \\
\hline$\kappa_{\text {Survey }}$ & 0.075432 & $0.009^{* * *}$ & 0.0657955 & 0.009157 * & 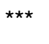 & 0.065464 & 0.007650 * & 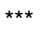 & 0.059189 & 0.008297 & $\star \star \star \star ~$ \\
\hline$\eta_{\text {Time }}$ & -0.00375 & 0.0022 * & -0.008059 & 0.0025314 * & 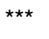 & -0.005065 & 0.001984 * & ** & -0.007672 & 0.001988 & 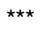 \\
\hline$\beta_{\text {LBLB }}$ & 0.011116 & 0.0305 & 0.106963 & 0.0321414 * & 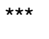 & -0.004376 & 0.027623 & & 0.083330 & 0.029121 & $\star \star \star \star ~$ \\
\hline$\beta_{\text {LBEQ }}$ & 0.012529 & 0.0079 & 0.0285687 & 0.0068408 * & 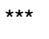 & 0.019656 & 0.006883 * & 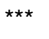 & 0.041006 & 0.005729 * & 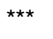 \\
\hline$\omega_{\text {LBPKM }}$ & -0.126063 & $0.0107^{* \star *}$ & -0.116246 & 0.0093441 * & 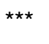 & -0.124200 & $0.009847^{*}$ & 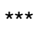 & -0.069951 & 0.010422 * & 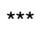 \\
\hline$\omega_{\text {EQPKM }}$ & -0.039599 & $0.0055^{* \star *}$ & -0.007066 & 0.0102923 & & -0.047478 & 0.005234 * & $\star \star \star *$ & -0.026919 & 0.008001 * & 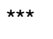 \\
\hline$\omega_{\text {EQTKM }}$ & 0.009174 & 0.0054 * & 0.0165605 & $0.0087077^{*}$ & & 0.019473 & 0.004737 * & $\star \star \star *$ & 0.032142 & 0.006497 * & 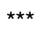 \\
\hline$\Psi_{\text {PKMKQ }}$ & 0.026149 & 0.0059 *** & 0.0572752 & 0.0098321 * & $\star \star \star ~$ & 0.020716 & 0.005365 * & $\star \star \star *$ & 0.059461 & 0.010168 * & *** \\
\hline$\Psi_{\text {TKMKQ }}$ & -0.042407 & $0.006^{\star \star \star}$ & -0.055671 & 0.009662 * & $\star \star \star$ & -0.042111 & 0.005864 * & $\star \star \star ~$ & -0.052318 & 0.009626 * & $\star \star \star ~$ \\
\hline$\phi_{\mathrm{EQKQ}}$ & 0.069316 & $0.0041^{* \star *}$ & 0.0329679 & 0.0040203 * & $* \star \star$ & 0.064134 & 0.004034 * & $* \star *$ & 0.025254 & 0.003103 * & $\star * \star *$ \\
\hline$\phi_{\text {LBTime }}$ & -0.006691 & $0.0016^{* \star *}$ & -0.002549 & 0.0017877 & & -0.007364 & 0.001440 * & $* \star *$ & -0.002763 & 0.001463 * & \\
\hline$\phi_{\text {LBQVmax }}$ & -0.085278 & $0.0154^{\star \star \star *}$ & -0.000473 & 0.0198758 & & -0.092689 & 0.013980 * & 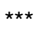 & -0.025189 & 0.021106 & \\
\hline$\lambda_{\text {QVTime }}$ & 0.000265 & 0.0011 & -0.001585 & 0.0012651 & & 0.002664 & 0.001110 * & $\star \star$ & -0.000779 & 0.001055 & \\
\hline$\lambda_{\text {QVQV }}$ & -0.065857 & $0.0095^{* \star *}$ & -0.01457 & 0.0117067 & & -0.095414 & 0.011989 * & $* \star \star$ & -0.025230 & 0.010638 * & ** \\
\hline$\kappa_{\text {DENS }}$ & 0.362203 & $0.0236^{\star \star \star}$ & 0.085646 & 0.0232515 * & $* \star \star$ & 0.310573 & 0.022072 * & 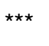 & 0.068347 & 0.020415 * & $\star \star \star ~$ \\
\hline$\kappa_{A G M}$ & -0.047503 & $0.0123 * \star \star *$ & 0.0341619 & 0.0112344 * & 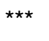 & 0.002379 & 0.011734 & & 0.050963 & $0.011255^{*}$ & $\star \star \star *$ \\
\hline$\eta_{\mathrm{Net}}$ & 0.34565 & $0.0469^{\star \star \star}$ & 0.270502 & 0.0522881 * & $\star \star \star$ & 0.374673 & 0.044781 * & $\star \star \star$ & 0.207331 & 0.049414 * & 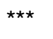 \\
\hline$\lambda_{\mathrm{KQKQ}}$ & -0.026572 & $0.0062 * \star \star$ & -0.014341 & 0.0075389 * & & -0.022529 & 0.006035 * & $\star \star \star$ & -0.010060 & 0.007511 & \\
\hline$\lambda=\sigma_{u} / \sigma_{v}$ & 2.04182 & $0.253^{\star \star \star}$ & 2.96542 & 0.35394 * & $\star \star \star$ & 1.146890 & 0.197689 * & $\star \star \star$ & 1.184720 & 0.182204 * & $\star \star \star ~$ \\
\hline$\sigma=\left(\sigma_{u}^{2}+\sigma_{u}^{2}\right)^{1 / 2}$ & 0.145822 & $0.0084^{* \star *}$ & 0.158192 & 0.0082829 * & 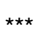 & 0.109170 & 0.009191 * & $\star \star \star ~$ & 0.110817 & 0.010076 * & 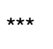 \\
\hline Log-likelihood & 473.629 & & 474.803 & & & 546.605 & & & 542.795 & & \\
\hline
\end{tabular}

Number of observations: 534

*** significant at the level of $1 \%$; ${ }^{* *}$ significant at the level of $5 \%$; ${ }^{*}$ significant at the level of $10 \%$.

The model that provides elasticities of substitution values with less variability is model II using the output set $\mathrm{Y}_{\text {II }}{ }^{11}$. The sample mean values of the own partial elasticities of substitution are for labour $\sigma_{\mathrm{LBLB}}=-1.10$, for transport equipment $\sigma_{\mathrm{EQEQ}}=-4.91$ and for materials $\&$ energy $\sigma_{\mathrm{EMEM}}=-0.32$ and the cross partial elasticities of substitution are $\sigma_{\mathrm{LBEQ}}=1.78, \sigma_{\mathrm{EQEM}}=0.41$ and $\sigma_{\mathrm{EMLB}}=0.11$. The cross partial elasticities of substitution mean values, with the exception of $\sigma_{\text {LBEQ }}$ (mean value of 
The external factor parameters estimates, such as weather and terrain, have, the expected positive signs and are statistically significant at the $1 \%$ probability level for the majority of models. This implies that countries with worse terrain and weather conditions have an increase in operational costs. Parameters related to population density (DENS) are all significant at the $1 \%$ probability level for each model. However, the elasticity of this variable is dependent on the output chosen. Likewise, the parameters related to the number of agglomeration cities (AGM) are mostly significant at the $1 \%$ probability level, but again vary considerably from model to model. The reason for the differences between model parameter values for the DENS and AGM variables can be explained by the fact that they have an implicit relationship with output which depends on the nature of the individual output measures used.

Two types of variables were introduced in the cost functions to explicitly characterise the quality of service effects: QVmax and the set of high speed dummy variables. For model II, the mean values of the cost elasticity of the quality of service QVmax are -0.05 and of -0.02 respectively for the output set given by $\mathrm{Y}_{\mathrm{I}}$ and $\mathrm{Y}_{\mathrm{II}}$. Thus, at the point of means, an increase in the quality of service is associated with an increase in operational costs. Regarding the results relating to the high speed dummy variables (HS220, DHS, TT, DTT), three main observations can be made. First, is that we do not find evidence of a significant effect on operational costs from the introduction of a high speed network: the elasticity associated with the DHS variable is statistically insignificant in all models. Second, the dummy variable which accounts for the increment of high speed usage 
(HS220) has a negative effect significant at the $1 \%$ probability level. Third, for the tilting train technology, gains on operational cost come not from the increment of traffic density of tilting trains technology but from impact of introducing this technology into the network.

\section{Estimates of technical and allocative inefficiencies}

In this sub-section sources of inefficiency are analysed using the cost structure results presented above. To avoid excessive and repetitive analysis we concentrate our discussion on the model that yields the most reliable measures for the railway cost structure based on the following criteria:

i. has the highest number of observations in the sample with correct (negative) estimated own elasticities of substitution signs;

ii. has non-negative measures of technical and allocative cost inefficiencies for all observations in the sample;

iii. has the highest maximum likelihood function estimate.

The model which best conforms to these criteria, for both aggregate output measures $\mathrm{Y}_{\mathrm{I}}$ and $\mathrm{Y}_{\text {II, }}$ is model II.

The estimates of the logarithmic cost inefficiency of the European railways was obtained according to each model's specification as a function of the maximum likelihood estimates of parameters and of the share residuals. Tables 4 reports the mean values of the logarithm of allocative cost inefficiency estimates together with their standard errors, the Wald test for the hypothesis that the set of parameters are jointly zero, and the respective probability values. 
The results shown in that table appear to indicate that European railways do generate excess costs as a result of allocative inefficient behaviour. Furthermore, the significant standard error of the groups mean and the probabilities values for the Wald test, show, in contrast to other studies such Gathon and Perelman (1992), Bosco (1996) or Parisio (1999), that there is also substantially different behaviour between firms with respect to the use of correct input proportion. The mean increase in variable costs due to allocative inefficiency is around $20 \%$ with output expressed as output revenue $\left(\mathrm{Y}_{\mathrm{I}}\right)$, with the lowest mean value of $1.9 \%$ for the VR/RHK firm and the highest value of $67 \%$ for DB. If output is measured as available output $\left(\mathrm{Y}_{\mathrm{II}}\right)$ the mean increase in variable costs due to allocative inefficiency is around $27 \%$ with the lowest mean firm value of $3.5 \%$ obtained for SJ/BV and the highest value of $93 \%$ for PKP. For both models with output set $\mathrm{Y}_{\mathrm{I}}$ and output set $\mathrm{Y}_{\text {II }}$, most of the mean firm values are significant at a probability level of 5\%. Thus, our results support the view that European railways bear additional cost because of they use inputs in the wrong proportions. 
Table 4 - Mean values of (log) allocative cost inefficiency

\begin{tabular}{|c|c|c|c|c|c|c|c|c|}
\hline \multirow{3}{*}{ Firms } & \multicolumn{8}{|c|}{ Model II } \\
\hline & \multicolumn{4}{|c|}{ Output $Y_{I}$} & \multicolumn{4}{|c|}{ Output $Y_{\text {II }}$} \\
\hline & Estimate & S.D. & $x^{2}$ & $\mathrm{P}$-value & Estimate & S.D. & $\chi^{2}$ & $P$-value \\
\hline $\mathrm{BR}$ & 0.3939 & 0.0782 & 25.38 & 0.00 & 0.4308 & 0.2137 & 4.06 & 0.04 \\
\hline $\mathrm{CD}$ & 0.0278 & 0.0093 & 8.83 & 0.00 & 0.1828 & 0.0181 & 102.20 & 0.00 \\
\hline CFF & 0.2246 & 0.0282 & 63.30 & 0.00 & 0.1502 & 0.1052 & 2.04 & 0.15 \\
\hline CFL & 0.0374 & 0.0383 & 0.95 & 0.33 & 0.1105 & 0.1433 & 0.59 & 0.44 \\
\hline $\mathrm{CH}$ & 0.1035 & 0.1311 & 0.62 & 0.43 & 0.1508 & 0.0593 & 6.46 & 0.01 \\
\hline CIE & 0.1313 & 0.0416 & 9.96 & 0.00 & 0.1311 & 0.1623 & 0.65 & 0.42 \\
\hline $\mathrm{CP}$ & 0.1903 & 0.0703 & 7.32 & 0.01 & 0.3114 & 0.1709 & 3.32 & 0.07 \\
\hline CP/REFER & 0.0415 & 0.0029 & 201.71 & 0.00 & 0.0427 & 0.0077 & 30.87 & 0.00 \\
\hline DB & 0.5145 & 0.0379 & 183.87 & 0.00 & 0.5109 & 0.0753 & 46.00 & 0.00 \\
\hline DB AG & 0.4246 & 0.0446 & 90.74 & 0.00 & 0.3286 & 0.0182 & 325.22 & 0.00 \\
\hline DSB & 0.0950 & 0.0462 & 4.22 & 0.04 & 0.0959 & 0.0227 & 17.83 & 0.00 \\
\hline EVR & 0.2428 & 0.0461 & 27.75 & 0.00 & 0.1522 & 0.0228 & 44.40 & 0.00 \\
\hline FS & 0.4879 & 0.1532 & 10.14 & 0.00 & 0.6001 & 0.2479 & 5.86 & 0.02 \\
\hline MAV & 0.0607 & 0.0192 & 10.04 & 0.00 & 0.3263 & 0.0119 & 753.15 & 0.00 \\
\hline NS & 0.1807 & 0.0415 & 19.00 & 0.00 & 0.0876 & 0.0448 & 3.82 & 0.05 \\
\hline NS BV/NV & 0.1186 & 0.0020 & 3684.20 & 0.00 & 0.0568 & 0.0031 & 341.92 & 0.00 \\
\hline NSB & 0.0411 & 0.0278 & 2.18 & 0.14 & 0.0572 & 0.0485 & 1.39 & 0.24 \\
\hline OBB & 0.0837 & 0.0557 & 2.26 & 0.13 & 0.1721 & 0.0397 & 18.83 & 0.00 \\
\hline PKP & 0.2954 & 0.0967 & 9.33 & 0.00 & 0.6567 & 0.0288 & 520.95 & 0.00 \\
\hline RENFE & 0.1876 & 0.0591 & 10.09 & 0.00 & 0.1932 & 0.1536 & 1.58 & 0.21 \\
\hline SJ & 0.1064 & 0.0611 & 3.03 & 0.08 & 0.1169 & 0.1502 & 0.61 & 0.44 \\
\hline SJBV & 0.0291 & 0.0158 & 3.40 & 0.07 & 0.0347 & 0.0122 & 8.07 & 0.00 \\
\hline SNCB & 0.1822 & 0.0555 & 10.79 & 0.00 & 0.2585 & 0.0759 & 11.60 & 0.00 \\
\hline SNCF & 0.3896 & 0.0362 & 115.94 & 0.00 & 0.2746 & 0.0788 & 12.16 & 0.00 \\
\hline SNCF/RFF & 0.3955 & 0.0111 & 1280.28 & 0.00 & 0.2563 & 0.0049 & 2683.52 & 0.00 \\
\hline $\mathrm{SZ}$ & 0.1168 & 0.0855 & 1.87 & 0.17 & 0.0445 & 0.0818 & 0.30 & 0.59 \\
\hline TCDD & 0.1374 & 0.1082 & 1.61 & 0.20 & 0.5966 & 0.1641 & 13.21 & 0.00 \\
\hline VR & 0.0796 & 0.0292 & 7.44 & 0.01 & 0.1433 & 0.1463 & 0.96 & 0.33 \\
\hline VR/RHK & 0.0184 & 0.0056 & 10.62 & 0.00 & 0.0655 & 0.0065 & 101.51 & 0.00 \\
\hline ZSR & 0.0441 & 0.0944 & 0.22 & 0.64 & 0.1899 & 0.1873 & 1.03 & 0.31 \\
\hline MEAN & 0.1894 & 0.1582 & 1.43 & 0.23 & 0.2418 & 0.2152 & 1.26 & 0.26 \\
\hline
\end{tabular}

Figures 1 and 2 show, for each firm, in successive five year periods (1972-75, 1976-80, 1981-85, 1986-90, 1991-95, 1996-99) the estimated mean percentage cost increase due to allocative inefficiency. 


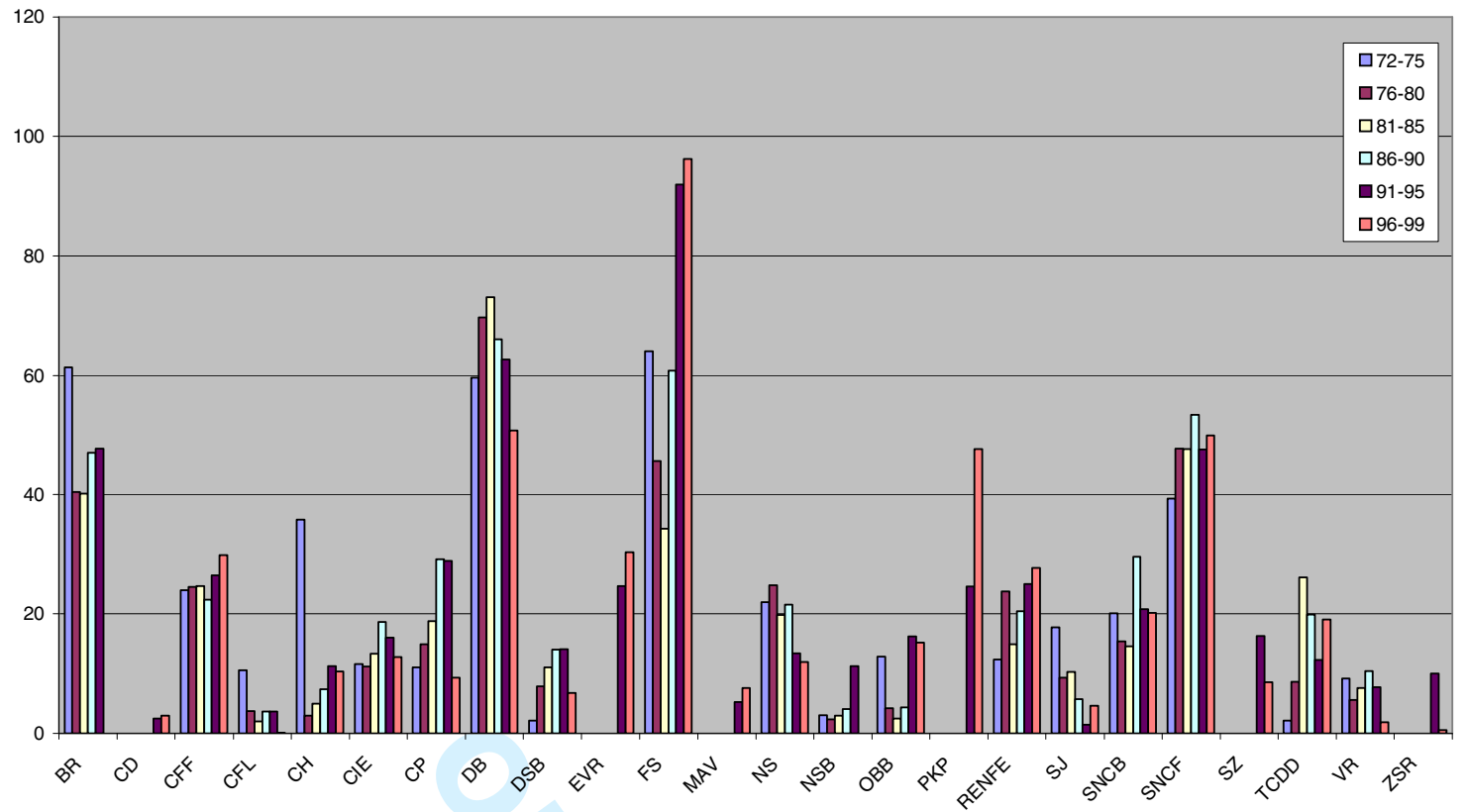

Figure 1 - Mean cost increase due to allocative efficiency (\%): Output $Y_{I}$

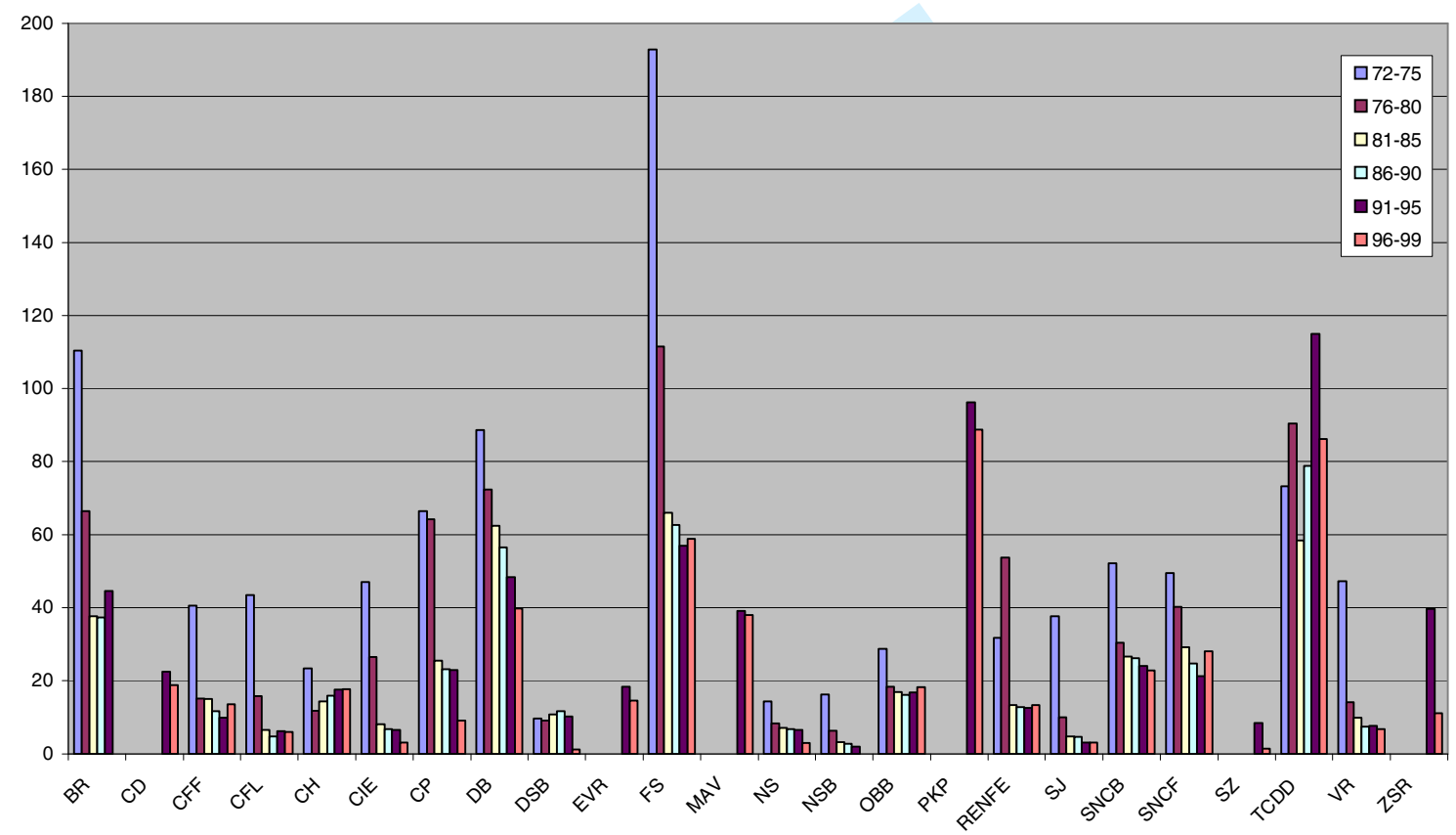

Figure 2 - Mean cost increase due to allocative efficiency (\%): Output $\mathbf{Y}_{\text {II }}$ 
When output is represented by train-kilometres (Figure 2), then with the exception of TCDD, all firms have decreased their allocative inefficiency during the sample period. However, if the demand effect is taking into account (Figure 1), then for FS, SNCF, RENFE, NSB and CP, we find over the same period that allocative inefficiency has often increased. In fact, over the sample period, under the pressure of large budget deficit, we know that all firms, with the exception of NS, have implemented internal management policy changes to reduce staff. This could explain the fall in allocative inefficiency shown in figure 2. The most likely reason that this fall is not also shown in figure 1 is that when we use a demand based measure of output we also capture effects that are outside the control of management and organisation restructuring and these have important impacts on 'global' allocative inefficiency. In fact this interpretation is consistent with the point of view of those who believe that the social benefits which railway transport provides are not compatible with objectives based exclusively on inefficiency reductions.

Table 5 reports the mean values of the estimates of technical inefficiency together with their standard errors, the Wald test for the hypothesis that the set of parameters are jointly zero, and the respective probability values. The estimated cost of technical inefficiency appears to be substantially lower than that associated with allocative inefficiency. From table 5, the mean increase in variable costs due to technical inefficiency is approximately $3.6 \%$ (in both output set alternatives). The lowest mean values are $1.9 \%$ (CP/ REFER) for output set $\mathrm{Y}_{\mathrm{I}}$ and $2.3 \%$ (NS BV/NV) with output measured by $\mathrm{Y}_{\mathrm{II}}$. The highest mean values are of $7.8 \%$ (SZ) and $8.3 \%$ (VR/RHK) respectively for output sets $Y_{I}$ and $Y_{\text {II. }}$ For both models, most of the mean firm values are significant at a probability level of $5 \%$. 


\section{Table 5 - Mean values of $(\log )$ technical cost inefficiency}

\begin{tabular}{|c|c|c|c|c|c|c|c|c|}
\hline \multirow{3}{*}{ Firms } & \multicolumn{8}{|c|}{ Model II } \\
\hline & \multicolumn{4}{|c|}{ Output $\mathrm{Y}_{\mathrm{I}}$} & \multicolumn{4}{|c|}{ Output $\mathrm{Y}_{\mathrm{II}}$} \\
\hline & Estimate & S.D. & $\chi^{2}$ & $\mathrm{P}$-value & Estimate & S.D. & $\chi^{2}$ & $\mathrm{P}$-value \\
\hline BR & 0.0297 & 0.0065 & 21.23 & 0.00 & 0.0344 & 0.0083 & 17.12 & 0.00 \\
\hline $\mathrm{CD}$ & 0.0193 & 0.0009 & 420.10 & 0.00 & 0.0326 & 0.0112 & 8.46 & 0.00 \\
\hline $\mathrm{CFF}$ & 0.0357 & 0.0076 & 22.06 & 0.00 & 0.0358 & 0.0073 & 23.86 & 0.00 \\
\hline CFL & 0.0340 & 0.0113 & 9.04 & 0.00 & 0.0338 & 0.0085 & 15.89 & 0.00 \\
\hline $\mathrm{CH}$ & 0.0377 & 0.0118 & 10.16 & 0.00 & 0.0373 & 0.0133 & 7.86 & 0.01 \\
\hline CIE & 0.0428 & 0.0151 & 8.03 & 0.00 & 0.0393 & 0.0155 & 6.41 & 0.01 \\
\hline $\mathrm{CP}$ & 0.0289 & 0.0132 & 4.79 & 0.03 & 0.0330 & 0.0109 & 9.10 & 0.00 \\
\hline CP/REFER & 0.0190 & 0.0036 & 27.51 & 0.00 & 0.0299 & 0.0051 & 33.88 & 0.00 \\
\hline DB & 0.0337 & 0.0124 & 7.45 & 0.01 & 0.0450 & 0.0189 & 5.70 & 0.02 \\
\hline DB AG & 0.0343 & 0.0100 & 11.88 & 0.00 & 0.0406 & 0.0135 & 9.03 & 0.00 \\
\hline DSB & 0.0317 & 0.0077 & 17.01 & 0.00 & 0.0287 & 0.0060 & 22.84 & 0.00 \\
\hline EVR & 0.0233 & 0.0210 & 1.23 & 0.27 & 0.0237 & 0.0041 & 32.75 & 0.00 \\
\hline FS & 0.0345 & 0.0126 & 7.48 & 0.01 & 0.0308 & 0.0129 & 5.68 & 0.02 \\
\hline MAV & 0.0241 & 0.0062 & 15.15 & 0.00 & 0.0366 & 0.0084 & 19.15 & 0.00 \\
\hline NS & 0.0381 & 0.0073 & 27.54 & 0.00 & 0.0445 & 0.0102 & 19.07 & 0.00 \\
\hline NS BV/NV & 0.0551 & 0.0012 & 2258.93 & 0.00 & 0.0229 & 0.0014 & 275.27 & 0.00 \\
\hline NSB & 0.0294 & 0.0086 & 11.60 & 0.00 & 0.0451 & 0.0226 & 3.99 & 0.05 \\
\hline OBB & 0.0243 & 0.0040 & 36.08 & 0.00 & 0.0262 & 0.0069 & 14.32 & 0.00 \\
\hline PKP & 0.0410 & 0.0232 & 3.13 & 0.08 & 0.0598 & 0.0475 & 1.58 & 0.21 \\
\hline RENFE & 0.0374 & 0.0198 & 3.56 & 0.06 & 0.0319 & 0.0102 & 9.81 & 0.00 \\
\hline SJ & 0.0411 & 0.0082 & 25.34 & 0.00 & 0.0333 & 0.0067 & 24.53 & 0.00 \\
\hline SJBV & 0.0454 & 0.0095 & 22.90 & 0.00 & 0.0452 & 0.0109 & 17.18 & 0.00 \\
\hline SNCB & 0.0353 & 0.0138 & 6.52 & 0.01 & 0.0304 & 0.0092 & 10.93 & 0.00 \\
\hline SNCF & 0.0365 & 0.0089 & 16.78 & 0.00 & 0.0314 & 0.0121 & 6.70 & 0.01 \\
\hline SNCF/RFF & 0.0549 & 0.0077 & 50.43 & 0.00 & 0.0313 & 0.0031 & 103.89 & 0.00 \\
\hline $\mathrm{SZ}$ & 0.0747 & 0.0266 & 7.87 & 0.01 & 0.0504 & 0.0131 & 14.90 & 0.00 \\
\hline TCDD & 0.0501 & 0.0323 & 2.41 & 0.12 & 0.0403 & 0.0194 & 4.33 & 0.04 \\
\hline VR & 0.0300 & 0.0116 & 6.67 & 0.01 & 0.0248 & 0.0098 & 6.39 & 0.01 \\
\hline VR/RHK & 0.0683 & 0.0050 & 183.24 & 0.00 & 0.0825 & 0.0041 & 404.06 & 0.00 \\
\hline ZSR & 0.0320 & 0.0073 & 19.17 & 0.00 & 0.0260 & 0.0031 & 71.66 & 0.00 \\
\hline MEAN & 0.0358 & 0.0163 & 4.82 & 0.03 & 0.0356 & 0.0157 & 5.15 & 0.02 \\
\hline
\end{tabular}

Figure 3 and 4 show, for each firm, in successive five years periods (1972-75, 1976-80, 1981-85, 1986-90, 1991-95, 1996-99) the estimated mean percentage cost increase due to technical inefficiency. 


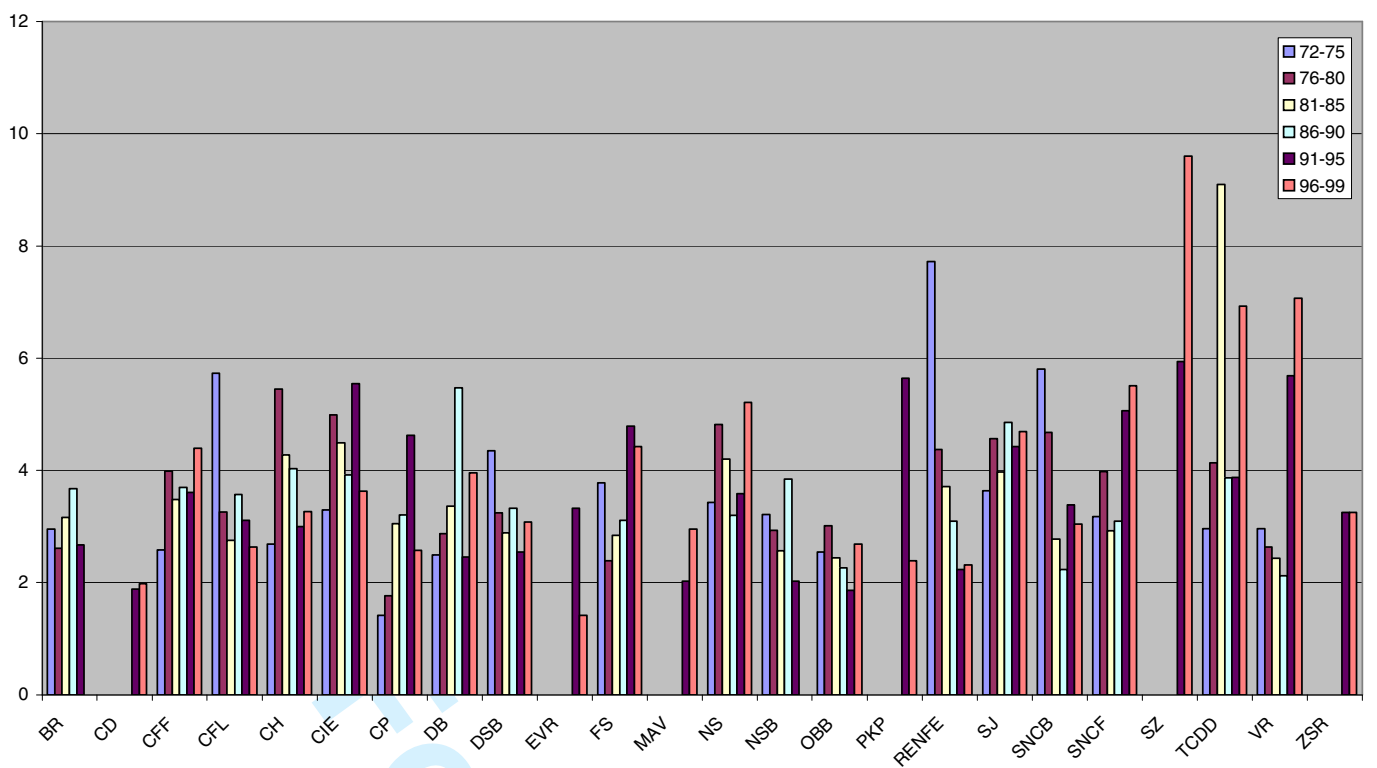

Figure 3 - Mean cost increase due to technical efficiency (\%): Output $Y_{I}$

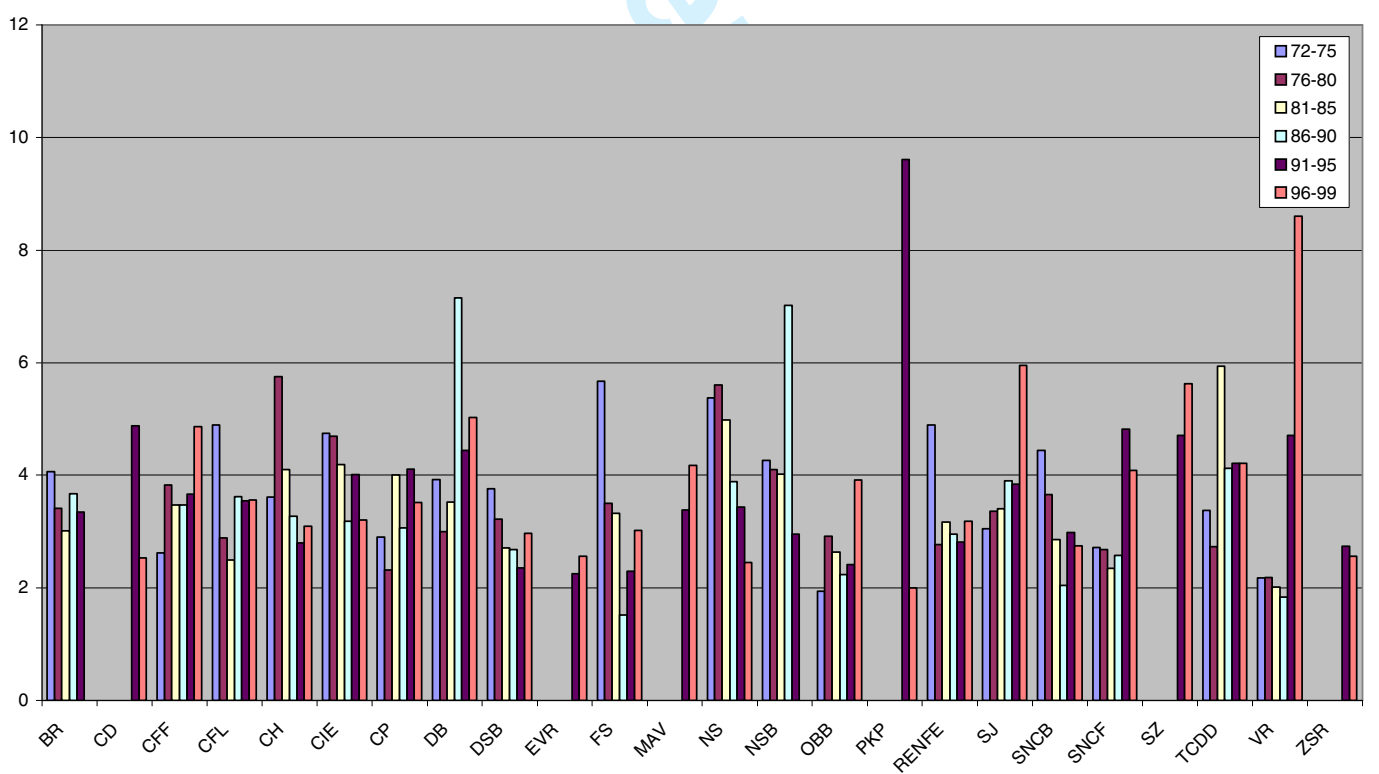

Figure 4 - Mean cost increase due to technical efficiency (\%): Output $Y_{\text {II }}$

The graphs show that there is a significant variability in the technical efficiency levels of firms over time. Furthermore, the table shows that there is no clear trends in the behaviour of firms: some 
show continuously decreasing technical efficiency values (e.g. RENFE, PKP and SNCB), others continuously increasing (e.g. VR, SZ, SNCF, SJ and CFF), while some show more erratic variation over time (e.g. TDCC, DB and NSB). Thus, we have mixed evidence across our sample of firms but despite this there are also some clear finding. First, the results do show that there are firms which, regardless of the output considered, perform well: these are VR and ÖBB. Second, we find that CD performs well if market demand is taken into account but not so well when only internal management is taken into account. Finally, we observe that the most poorly performing firms tend to be those that have separated infrastructure from operational services and which actually show higher technical inefficiency after the separation has taken place. The best example of this effect is VR and VR/RHK, which performed very well until the separation, after which it became the least efficient railway in the sample.

Table 6 shows a comparison of our results with those of analogous studies using similar data, by providing a ranking of the technical inefficiency values obtained for each railway. Correlations among ranking results are generally low, with the exception of our model II ( $\mathrm{Y}_{\text {II }}$ ) and those of Parisio (1999) which gives a rank correlation score of 0.70. In fact it is very difficult to undertake a comparison of results across studies due to the different modelling techniques applied, not least, the fact that in some studies estimates are derived using a production function framework which does not take into account allocative inefficiencies. 
Table 6 - Ranking in terms of technical inefficiency in distinct studies

\begin{tabular}{|c|c|c|c|c|c|c|c|c|c|}
\hline \multirow[b]{2}{*}{ Firms } & \multirow{2}{*}{$\begin{array}{l}\text { Gathon and } \\
\text { Perelman (1992) }\end{array}$} & \multirow{2}{*}{$\begin{array}{l}\text { Perelman and } \\
\text { Pestieau (1988) }\end{array}$} & \multirow{2}{*}{$\begin{array}{c}\text { Coelli and } \\
\text { Perelman (1999) }\end{array}$} & \multirow{2}{*}{$\begin{array}{c}\text { Coelli and } \\
\text { Perelman }(2000) \\
\end{array}$} & \multirow{2}{*}{$\begin{array}{c}\text { Gathon and } \\
\text { Pestieau (1995) }\end{array}$} & \multirow{2}{*}{$\begin{array}{l}\text { Deprins and } \\
\text { Simar (1988) } \\
\end{array}$} & \multirow{2}{*}{$\begin{array}{l}\text { Parisio } \\
\text { (1999) } \\
\end{array}$} & \multicolumn{2}{|c|}{ Present study } \\
\hline & & & & & & & & Model II $\left(\mathrm{Y}_{\mathrm{I}}\right)$ & $\operatorname{Model~II~}\left(\mathrm{Y}_{\mathrm{II}}\right)$ \\
\hline$\overline{\mathrm{BR}}$ & 15 & 13 & 5 & 6 & 4 & 16 & 6 & 4 & 11 \\
\hline CFF & 14 & 10 & 2 & 14 & 3 & 3 & 7 & 11 & 12 \\
\hline CFL & 17 & 15 & 10 & 8 & 16 & 14 & & 8 & 10 \\
\hline $\mathrm{CH}$ & 12 & 18 & 11 & 17 & 15 & 7 & & 14 & 13 \\
\hline $\mathrm{CIE}$ & 16 & 1 & 4 & 2 & 6 & 2 & & 17 & 14 \\
\hline $\mathrm{CP}$ & 6 & 6 & 16 & 10 & 7 & 10 & & 2 & 8 \\
\hline DB & 11 & 14 & 8 & 5 & 13 & 13 & 8 & 7 & 17 \\
\hline DSB & 4 & 3 & 15 & 11 & 18 & 9 & & 6 & 3 \\
\hline FS & 19 & 17 & 17 & 7 & 9 & 12 & 1 & 9 & 5 \\
\hline NS & 2 & 2 & 1 & 1 & 1 & 1 & 4 & 15 & 16 \\
\hline NSB & 7 & 4 & 5 & 3 & 17 & 17 & & 3 & 18 \\
\hline OBB & 18 & 11 & 9 & 16 & 14 & 6 & 2 & 1 & 2 \\
\hline RENFE & 13 & 12 & 13 & 13 & 11 & 11 & & 13 & 7 \\
\hline SJ & 5 & 8 & 14 & 16 & 8 & 8 & & 16 & 9 \\
\hline SNCB & 9 & 16 & 12 & 4 & 12 & 18 & 5 & 10 & 4 \\
\hline SNCF & 2 & 7 & 3 & 9 & 5 & 4 & 3 & 12 & 6 \\
\hline TCDD & 7 & 19 & & & 2 & 15 & & 18 & 15 \\
\hline $\mathrm{VR}$ & 1 & 9 & 7 & 2 & 10 & 5 & & 5 & 1 \\
\hline
\end{tabular}

In fact, the main difference between our results and those of previous studies relates to the relative magnitude of the estimates of technical and allocative efficiency. We find a much greater role for allocative inefficiency in generating cost increases than technical inefficiency. Thus, while our results do support the common view that European railways bear additional costs due to inefficiencies, in contrast to previous studies, we find that these inefficiencies arise to a larger extent because of wrong input proportions than equiproportionate input waste.

As mentioned previously, our technical inefficiency results tend to be lower in magnitude than those of previous studies. We believe this is mainly due to differences in models employed, especially since some models are based on production function estimation which does not take into account allocative inefficiency effects. Of course allocative inefficiency results themselves are also very sensitive to methodology, and this is shown in the Kumbhakar studies of US railways (Kumbhakar 1988a, 1998b, 1989). However, as mentioned above, analyses by Bosco (1996) and Parisio (1999) do generate allocative inefficiency results which are very similar to our estimates providing some consistent evidence of the magnitude of this component. 


\section{The determinants of inefficiency}

In order to identify the determinants of inefficiency we regress the aggregate inefficiency scores on a set of explanatory variables. From the literature, we find a set of such variables that are commonly used.

i. Capacity utilisation - the average passenger load per train (PLOAD) and the average freight load per train (FLOAD) are used as indices of train utilisation.

ii. Quality of services - the index variable for quality of service (QVmax) characterises operational improvements either through higher traffic flows or transport infrastructure and equipment improvements (such as the percentage of double track and of electrified lines, frequency and average maximum velocity of tractive stock).

iii. Subsidy - the policy subsidy variable (SUB_VC) measures the ratio of subsidy to operating costs. Subsidy policy should ideally be examined according to the types of subsidies and the way in which they are provided, however, due to limited information we use the level of aggregate subsidy only.

iv. Firm capacity and autonomy - the network length (NET) is used here as a proxy for firm capacity and degree of firm autonomy. Firms with higher autonomy are expect to be able to adjust their network length more easily than firms conditioned to conserve lines considered socially desirable but economically unprofitable. 
v. Managerial autonomy - in addition to the network length variable the percentage of passenger train-kilometres in total train-kilometres (PERCP) is included as an indicator of managerial operational autonomy. Firms with higher autonomy are more able to adjust their supply of passenger services than firms with higher government control.

vi. Over-employment and union control - the number of employees per train-kilometres (STAFF) is included in an attempt to characterise firms with over-employment . It could also provide a proxy for the power of union forces and government control.

In addition to these variables we also wish to test for the effects of high speed technology and vertical separation on cost efficiency. Accordingly, we include in the regression high speed dummy variables (DHS and DTT) and a dummy variable identifying the organisational implementation of vertical separation policy reform (DSEP).

The regression of cost inefficiency, with dependent variable given by the sum of logarithm of the cost of technical inefficiency and the logarithm of the cost of allocative inefficiency, was estimated using a three step Generalised Least Square (GLS) estimator for time series cross section (TSCS) data $^{12}$. To ease the interpretation of results, the independent variables were expressed also in logs. In line with the cost function estimation, two alternative regression models were run, one in which the dependent variable is derived from model II of cost function estimation with the output measured by the revenue output $\left(\mathrm{Y}_{\mathrm{I}}\right)$; and one in which the dependent variable is derived from model II with output expressed by train-kilometres ( $\left.\mathrm{Y}_{\text {II }}\right)$. The results are shown in table 7. 


\section{Table 7 - The determinants of inefficiency: regression results}

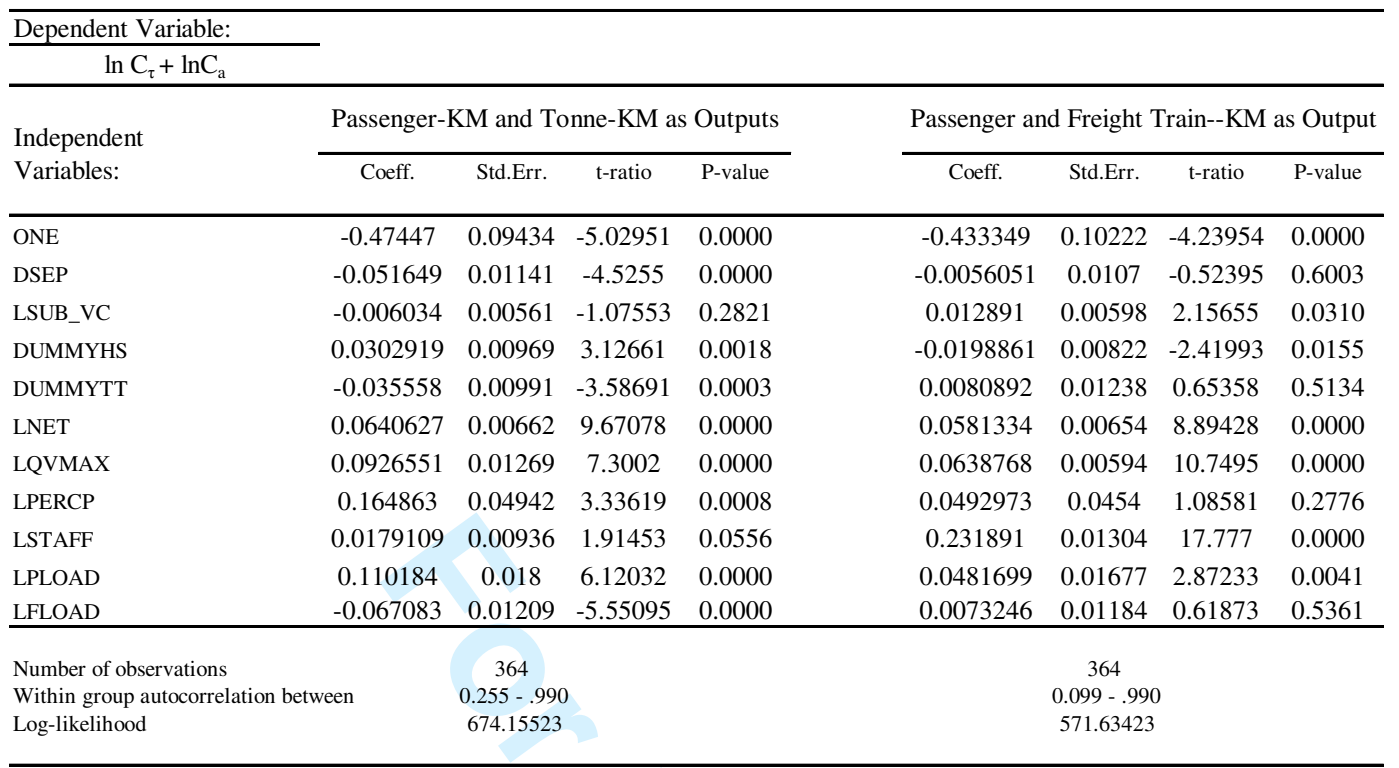

The ratio of subsidy to the total operating expenses has a statistically significant positive coefficient with output given by $\mathrm{Y}_{\mathrm{II}}$ but not by $\mathrm{Y}_{\mathrm{I}}$. This may indicate that subsidisation provides little incentive to the firm to improve productivity or minimise costs. However, if the demand response is taken into account then perhaps any loss of efficiency is balanced by other particular types of subsidies, such as ticket price and conditional subsidies, which yield demand increases and thus nominal efficiency gains.

The vertical separation of infrastructure and operations (DSEP) appears to increase efficiency. However, this increase is only statistically significant in the first model which is based on revenue output. This policy allows for efficiency cost savings of 5\%, mainly through an improved adjustment of inputs used and revenue outputs, but the gains in cost efficiency from internal production process are actually less. It is important to emphasise here that results presented earlier in this paper showed an increase in cost due to technical inefficiency provoked by vertical separation So in fact, overall the results shown in table 7 suggest that the gains in allocative 
efficiency provided by this structural reform overcome the loss in terms of technical efficiency estimated earlier in the paper.

The variables characterising the potential production capacity of firms, such as NET and QVmax, have a statistically significant positive coefficient in both models $\left(\mathrm{Y}_{\mathrm{I}}\right)$ and $\left(\mathrm{Y}_{\mathrm{II}}\right)$. Regarding the network variable, we might expect excess capacity to arise due to the possible existence of network branches with low demand. For the variable QVmax, the positive coefficient results indicate that any inherent cost benefits associated with improved quality are not enough to overcome the negative effect on efficiency due to an excess of the capacity of transport capital stock.

Concentrating on the results from the first model (using $\mathrm{Y}_{\mathrm{I}}$ ), the variables PERCP and PLOAD have statistically significant positive coefficients which indicate substantial increases in cost inefficiency from greater concentration in passenger services. Note also that the coefficient of FLOAD is statistically significant but with a negative value. The most likely explanation for these results is that passenger services, which have more visible social implications than freight services, are less prone to traffic reduction through governmental policies or transport authority regulators than freight service. Consequently, the inefficiencies induced by excess service capacity arise more from passenger services than freight services, which have undergone more significant adjustments over time. However, it should be stressed, by other side, that this negative coefficient may simply indicate that heavily loaded freight railways are closest to the cost frontier.

\footnotetext{
Regarding staff usage, the positive coefficient is indicative of over-employment, but its contribution to inefficiency costs seems to be of little importance when compared with the other variables just described.
} 


\begin{abstract}
We have concentrated much of our discussion on the first model (using $\mathrm{Y}_{\mathrm{I}}$ ), in which cost inefficiencies can be described as 'global' since they includes sources of inefficiency that are beyond managerial control including influences from public policy on the capacity supplied and on the output consumed. In the second model (using $\mathrm{Y}_{\mathrm{II}}$ ), any excess capacity which arises from the operation of low demand network branches does not feature in the measurement of cost inefficiencies. Thus, we would expect that the determinants of cost efficiencies to be different than those estimated for the first model, having a more specific association with managerial practices.
\end{abstract}

In fact, as expected the second model results do show that the variables related to excess capacity, such as NET and QVmax, have statistically significant coefficients but produce a much a lower effect on inefficiency. The most important difference between models I and II, however, are associated with the variables describing operational behaviour. The percentage of passenger train$\mathrm{kms}$ in total train-kms as well as the average freight load per train are not statistically significant, and in contrast to the first model, do not make a contribution to cost inefficiency. Even the average passenger load per train, while statistically significant in model II, makes a contribution to inefficiency that is less than a half that shown in the first model. The average staff per train-km variable has a statistically significant positive coefficient and its elasticity (0.23) indicates a relatively high degree of importance in giving rise to cost inefficiency. Thus, when demand factors are excluded from output measurement, cost inefficiencies are particularly sensitive to overemployment, with factors such as intensive train occupation and heavier concentration in passenger services having a lower impact. 
Regarding the effect of high speed technology, in the first model using $\mathrm{Y}_{\mathrm{I}}$ as output, both traditional high speed and tilting train technology coefficients are statistically significant; however, traditional high speed technology contributes to an increase in inefficiency costs and tilting train technology to a reduction of inefficiency costs. If at first glance these are surprising results, they are in conformity with what have been said in relation to other variable impacts. Traditional high speed technology involves a substantially higher investment in capital stock than tilting train technology and also requires new lines. Thus, it is not surprising that in presence of networks with excess capacity, high speed technology contributes to an increase in cost inefficiency; while tilting train technology, which uses the existent network, contributes to a reduction of cost inefficiency. It is, however, important to emphasise that traditional high speed technology is not per se the cause of cost inefficiency increases, but is due rather to the inherent excess of capacity produced by its introduction. In relation to the introduction of tilting train technology our estimates indicate that this gives rise to a decrease in cost inefficiencies of around $3.5 \%$.

Considering the second model based on output $\mathrm{Y}_{\mathrm{II}}$, in which cost inefficiencies are measured taking into account only the available output, the tilting train technology coefficient is not statistically significant. This means that the type of service supplied by this technology does not differ substantially in its operational characteristics from usual railway transport services, and thus, it does not have additional impact on cost inefficiencies. In the case of traditional high speed technology we find that its introduction allows for a reduction of cost inefficiencies of around $2 \%$. This confirms our hypothesis that if the excess capacity supplied it is not taken into account in the measure of inefficiency, then high speed technology serves to improves efficiency. 
To summarise, we find that cost inefficiencies can essentially be explained by the excess of capacity supplied and by the discrepancies resulting from an over-employment of labour with respect to the best practice level. Over-employment is clearly indicated by the high and positive residuals of the labour input cost share equation. These explanations are further substantiated by the fact that larger firms located in developed countries, where union labour forces tend to be more successful in demanding better salaries and where there are large networks with higher investment in capital stock improvements, also tend to be those that show high levels of allocative inefficiency.

\section{A decomposition of TFP growth}

In this final results section we present measures of TFP growth for our railway firms and decompose the sources of TFP into those derived from scale economies, technological progress, gains in efficiency, and changes in quality of service and network characteristics. To do so, we first have to estimate economies of scale and technological progress.

We estimate economies of scale from the expression ${ }^{13}$ :

$$
R T D=\frac{1-\sum_{k} \varepsilon_{K_{k}}^{V C}}{\sum_{j} \varepsilon_{Y_{j}}^{V C}}
$$

where $\varepsilon^{\mathrm{VC}} \mathrm{Yj}$ is the elasticity of variable costs with respect to output $\mathrm{j}$ and $\varepsilon_{\mathrm{K}}{ }^{\mathrm{VC}}$ is the elasticity of variable cost with respect to quasi-fixed infrastructure capital stock, calculated for the long-run equilibrium values of the quasi-fixed input factor. These optimal levels of the fixed factor could be derived from the long-run envelope function of the short-run total cost functions ${ }^{14}$.

The mean firm values of economies of scale estimates together with their standard errors, the Wald test for the hypothesis that there are constant returns to scale, and respective probability values are 
shown in table 8 . The results show, as expected and consistent with the majority of previous studies, increasing returns to traffic density for all firms ${ }^{15}$.

Table 8 - Mean values of returns to scale (economies of density)

\begin{tabular}{|c|c|c|c|c|c|c|c|c|}
\hline \multirow{3}{*}{ Firms } & \multicolumn{8}{|c|}{ Model II } \\
\hline & \multicolumn{4}{|c|}{ OUTPUT $Y_{\text {I }}$} & \multicolumn{4}{|c|}{ OUTPUT $Y_{\text {II }}$} \\
\hline & Estimate & S.D. & $\chi^{2}$ & $\mathrm{P}$-value & Estimate & S.D. & $\chi^{2}$ & P-value \\
\hline $\mathrm{BR}$ & 3.3813 & 0.7126 & 11.17 & 0.00 & 1.6674 & 0.0910 & 53.74 & 0.00 \\
\hline $\mathrm{CD}$ & 2.2464 & 0.3108 & 75489.96 & 0.00 & 1.5570 & 0.0468 & 461408.96 & 0.00 \\
\hline $\mathrm{CFF}$ & 4.7381 & 0.8019 & 21.73 & 0.00 & 1.9228 & 0.0881 & 109.84 & 0.00 \\
\hline CFL & 5.5240 & 2.3498 & 3.71 & 0.05 & 2.1652 & 0.2244 & 26.95 & 0.00 \\
\hline $\mathrm{CH}$ & 3.0008 & 0.8609 & 5.40 & 0.02 & 1.7303 & 0.1143 & 40.85 & 0.00 \\
\hline CIE & 2.6900 & 0.3679 & 21.10 & 0.00 & 1.8824 & 0.1192 & 54.81 & 0.00 \\
\hline $\mathrm{CP}$ & 2.6398 & 0.3571 & 21.09 & 0.00 & 1.7819 & 0.1537 & 25.87 & 0.00 \\
\hline CP/REFER & 2.8468 & 0.1316 & 196.81 & 0.00 & 1.7411 & 0.0024 & 96261.01 & 0.00 \\
\hline DB & 5.2251 & 0.6415 & 43.37 & 0.00 & 1.8386 & 0.0293 & 817.33 & 0.00 \\
\hline DB AG & 3.3206 & 0.3483 & 44.38 & 0.00 & 1.6344 & 0.0346 & 335.44 & 0.00 \\
\hline DSB & 3.3549 & 0.4541 & 26.89 & 0.00 & 1.7244 & 0.0285 & 645.07 & 0.00 \\
\hline EVR & 1.8700 & 0.0919 & 89.71 & 0.00 & 1.7557 & 0.1217 & 38.54 & 0.00 \\
\hline FS & 3.1251 & 0.9423 & 5.09 & 0.02 & 1.7180 & 0.1006 & 50.93 & 0.00 \\
\hline MAV & 2.3304 & 0.1664 & 63.92 & 0.00 & 1.5301 & 0.0101 & 2774.70 & 0.00 \\
\hline NS & 4.9668 & 1.0954 & 13.11 & 0.00 & 1.7732 & 0.1180 & 42.96 & 0.00 \\
\hline NS BV/NV & 2.5550 & 0.0096 & 26098.45 & 0.00 & 1.5263 & 0.0322 & 267.26 & 0.00 \\
\hline NSB & 4.0007 & 0.5142 & 34.05 & 0.00 & 1.9088 & 0.0966 & 88.51 & 0.00 \\
\hline OBB & 3.1794 & 0.7321 & 8.86 & 0.00 & 1.7313 & 0.0993 & 54.21 & 0.00 \\
\hline PKP & 2.3953 & 0.3918 & 12.68 & 0.00 & 1.5531 & 0.0382 & 209.35 & 0.00 \\
\hline RENFE & 3.3372 & 0.6604 & 12.53 & 0.00 & 1.8000 & 0.0960 & 69.49 & 0.00 \\
\hline SJ & 5.7732 & 2.1398 & 4.98 & 0.03 & 2.0216 & 0.1608 & 40.38 & 0.00 \\
\hline $\mathrm{SJ} / \mathrm{BV}$ & 3.3590 & 0.4294 & 30.18 & 0.00 & 1.7991 & 0.0593 & 181.73 & 0.00 \\
\hline SNCB & 3.8575 & 1.0659 & 7.19 & 0.01 & 1.7904 & 0.0665 & 141.31 & 0.00 \\
\hline SNCF & 3.7453 & 0.3355 & 66.96 & 0.00 & 1.7507 & 0.0573 & 171.83 & 0.00 \\
\hline SNCF/RFF & 2.8820 & 0.0742 & 643.82 & 0.00 & 1.6627 & 0.0059 & 12517.80 & 0.00 \\
\hline $\mathrm{SZ}$ & 2.3866 & 0.2572 & 29.08 & 0.00 & 1.7593 & 0.1002 & 57.47 & 0.00 \\
\hline TCDD & 1.6785 & 0.3848 & 3.11 & 0.08 & 1.8502 & 0.1998 & 18.11 & 0.00 \\
\hline VR & 3.9397 & 0.9142 & 10.34 & 0.00 & 1.9684 & 0.1054 & 84.47 & 0.00 \\
\hline VR/RHK & 3.2154 & 0.0538 & 1694.46 & 0.00 & 1.8493 & 0.0249 & 1164.31 & 0.00 \\
\hline ZSR & 2.0895 & 0.1231 & 78.26 & 0.00 & 1.5929 & 0.1002 & 35.00 & 0.00 \\
\hline MEAN & 3.5592 & 1.4097 & 3.30 & 0.07 & 1.8074 & 0.1760 & 21.05 & 0.00 \\
\hline
\end{tabular}

To calculate technological progress we measure the common rate at which all input can be decreased over time with outputs held fixed. We denote this quantity PGX and introduce it directly into the estimation of TFP growth rate. Thus, technical progress is given by: 


$$
P G X=-\left(\partial \ln g^{V} / \partial t\right) /\left(1-\sum_{k}\left(\partial \ln g^{V} / \partial \ln K_{k}\right)\right)
$$

where $K$ denotes the quasi-fixed factors, $g^{\mathrm{V}}$ the variable cost function and $y_{j}$ the outputs. Following Morrison (1986), Morrison (1985) and Oum et al. (1991), the values of PGX were calculated using the long-run equilibrium values of quasi-fixed inputs.

Table 9 shows values of PGX estimated from model II. Results from these specifications are quite similar under either output set (using $\mathrm{Y}_{\mathrm{I}}$ or $\mathrm{Y}_{\mathrm{II}}$ ), with sample mean values of 0.0156 and 0.0136 respectively. The Wald test for the hypothesis that the set of firm's PGX estimates are jointly zero is rejected at the $1 \%$ probability level for all firms, except for CD and PKP in the model using $\mathrm{Y}_{\mathrm{I}}$ as output set. The Wald test for the hypothesis that the sample set's PGX estimates are jointly zero is rejected at the $1 \%$ of probability level for both models. 
Table 9 - Means value of technical progress (PGX)

\begin{tabular}{|c|c|c|c|c|c|c|c|c|}
\hline \multirow{3}{*}{ Firms } & \multicolumn{8}{|c|}{ Model II } \\
\hline & \multicolumn{4}{|c|}{ OUTPUT Y $_{I}$} & \multicolumn{4}{|c|}{ OUTPUT $_{\text {II }}$} \\
\hline & Estimate & S.D. & $\bar{\chi}^{2}$ & $P$-value & Estimate & S.D. & $\chi^{2}$ & $P$-value \\
\hline BR & 0.0128 & 0.0024 & 28.93 & 0.00 & 0.0147 & 0.0012 & 140.59 & 0.00 \\
\hline $\mathrm{CD}$ & 0.0064 & 0.0036 & 3.14 & 0.08 & 0.0096 & 0.0015 & 43.20 & 0.00 \\
\hline $\mathrm{CFF}$ & 0.0179 & 0.0016 & 127.20 & 0.00 & 0.0164 & 0.0009 & 350.11 & 0.00 \\
\hline CFL & 0.0204 & 0.0052 & 15.20 & 0.00 & 0.0161 & 0.0016 & 103.47 & 0.00 \\
\hline $\mathrm{CH}$ & 0.0235 & 0.0038 & 37.47 & 0.00 & 0.0123 & 0.0021 & 33.61 & 0.00 \\
\hline CIE & 0.0149 & 0.0022 & 48.25 & 0.00 & 0.0122 & 0.0009 & 174.19 & 0.00 \\
\hline $\mathrm{CP}$ & 0.0170 & 0.0022 & 62.33 & 0.00 & 0.0127 & 0.0007 & 313.17 & 0.00 \\
\hline CP/REFER & 0.0146 & 0.0007 & 485.59 & 0.00 & 0.0137 & 0.0002 & 7715.75 & 0.00 \\
\hline DB & 0.0182 & 0.0013 & 193.57 & 0.00 & 0.0158 & 0.0006 & 806.47 & 0.00 \\
\hline DB AG & 0.0120 & 0.0017 & 48.97 & 0.00 & 0.0141 & 0.0006 & 567.45 & 0.00 \\
\hline DSB & 0.0164 & 0.0023 & 49.43 & 0.00 & 0.0146 & 0.0012 & 154.93 & 0.00 \\
\hline EVR & 0.0047 & 0.0010 & 22.80 & 0.00 & 0.0063 & 0.0004 & 255.01 & 0.00 \\
\hline FS & 0.0123 & 0.0038 & 10.45 & 0.00 & 0.0136 & 0.0018 & 56.99 & 0.00 \\
\hline MAV & 0.0090 & 0.0018 & 25.66 & 0.00 & 0.0108 & 0.0009 & 154.78 & 0.00 \\
\hline NS & 0.0192 & 0.0041 & 21.84 & 0.00 & 0.0178 & 0.0013 & 175.85 & 0.00 \\
\hline NS BV/NV & 0.0078 & 0.0001 & 3154.72 & 0.00 & 0.0145 & 0.0003 & 2691.98 & 0.00 \\
\hline NSB & 0.0226 & 0.0029 & 60.43 & 0.00 & 0.0144 & 0.0007 & 380.21 & 0.00 \\
\hline OBB & 0.0123 & 0.0041 & 9.00 & 0.00 & 0.0128 & 0.0018 & 50.95 & 0.00 \\
\hline PKP & 0.0062 & 0.0029 & 4.42 & 0.04 & 0.0104 & 0.0014 & 58.65 & 0.00 \\
\hline RENFE & 0.0158 & 0.0017 & 83.77 & 0.00 & 0.0138 & 0.0013 & 104.80 & 0.00 \\
\hline SJ & 0.0227 & 0.0030 & 55.50 & 0.00 & 0.0156 & 0.0009 & 320.90 & 0.00 \\
\hline SJBV & 0.0140 & 0.0028 & 25.08 & 0.00 & 0.0134 & 0.0011 & 142.25 & 0.00 \\
\hline SNCB & 0.0135 & 0.0030 & 20.44 & 0.00 & 0.0149 & 0.0015 & 96.89 & 0.00 \\
\hline SNCF & 0.0143 & 0.0013 & 121.33 & 0.00 & 0.0141 & 0.0007 & 357.74 & 0.00 \\
\hline SNCF/RFF & 0.0087 & 0.0005 & 342.09 & 0.00 & 0.0126 & 0.0003 & 2534.57 & 0.00 \\
\hline SZ & 0.0100 & 0.0004 & 550.30 & 0.00 & 0.0112 & 0.0002 & 4113.74 & 0.00 \\
\hline TCDD & 0.0093 & 0.0020 & 21.95 & 0.00 & 0.0084 & 0.0012 & 45.29 & 0.00 \\
\hline VR & 0.0210 & 0.0017 & 152.70 & 0.00 & 0.0139 & 0.0011 & 172.14 & 0.00 \\
\hline VR/RHK & 0.0157 & 0.0006 & 710.97 & 0.00 & 0.0131 & 0.0003 & 2593.86 & 0.00 \\
\hline ZSR & 0.0041 & 0.0013 & 9.43 & 0.00 & 0.0090 & 0.0006 & 214.54 & 0.00 \\
\hline MEAN & 0.0156 & 0.0055 & 7.94 & 0.00 & 0.0136 & 0.0027 & 26.26 & 0.00 \\
\hline
\end{tabular}

Using output measure $\mathrm{Y}_{\mathrm{I}}$, we find that Eastern European firms together with TDCC and SNCF/RFF are those that show the worst performances with values less than $1 \%$ while the firms with values higher than $2 \%$ are CFL, CH, VR and SJ. When train-kilometres are used as output, technical progress (PGX) shows more homogeneous values among firms and only CD, EVR, TCDD and ZSR show values below $1 \%$, NS shows the highest mean productivity growth with a value of $1.78 \%$. 
Having estimated the movements of the cost function due to technical progress, the movements throughout the cost function due to economies of scale, the firms' efficiencies and the quality of service and network effects on cost function, the next step is to estimate the rate of growth of total factor productivity. To do so we follow the methodology proposed by Bauer (1990a) which gives a pure measure of TFP growth defined as:

$$
\dot{T F P}=\left[1-\frac{1}{R T D}\right] \dot{y}^{c}+T \dot{A} E \cdot\left(\frac{1}{1-\varepsilon_{c, K}}\right)-\sum_{m} \varepsilon_{c, q_{m}} \cdot\left(\partial q_{m} / \partial t\right) \cdot\left(\frac{1}{1-\varepsilon_{c, K}}\right)-P G X
$$

where, $\dot{y}^{c}=\sum_{j}\left[\frac{\varepsilon_{c, y_{j}}}{\sum_{j} \varepsilon_{c, y_{j}}}\right] \dot{y}_{j}$, RTD is the index of economies of scale, $\dot{y}_{j}$ is the annual rate of change of output $\mathrm{j}, \varepsilon_{c, y_{j}}$ is the cost elasticity of output $\mathrm{j}, T \dot{A} E$ the annual rate of change of cost efficiency, $\varepsilon_{c, K}$ is the capital stock infrastructure cost elasticity, $\varepsilon_{c, q_{m}}$ is the quality of service and network characteristics cost elasticities ${ }^{16}$, and PGX is the time rate of change of technological progress.

Table 10 shows the annual growth rate of TFP, as well as a decomposition of this growth into the contributions of gains in efficiency (TEA), of economies of scale (ES), of changes in quality of service and network characteristics (QN), and of technological progress (PGX). We can observed that, taking an average of all companies, TFP growth has taken place at an annual rate of $1.84 \%$ and $2.59 \%$ respectively for outputs measured as passenger(tonne)-kilometres ( $\mathrm{Y}_{\mathrm{I}}$ ) and passenger(freight) train-kilometres ( $\left.\mathrm{Y}_{\mathrm{II}}\right)$. These rates were mostly due to technological progress (1.39\% and $1.32 \%$ respectively for $\mathrm{Y}_{\mathrm{I}}$ and $\left.\mathrm{Y}_{\mathrm{II}}\right)$, and to a less degree due to gains in efficiency $(1.08 \%)$ if output is measured in train-kilometres and due to gains from quality of service and 
network characteristics improvements $(0.59 \%)$ if output is measured in passenger(tonne)kilometres.

Table 10 - A decomposition of TFP growth

\begin{tabular}{|c|c|c|c|c|c|c|c|c|c|c|}
\hline \multirow{2}{*}{ Firms } & \multicolumn{5}{|c|}{ Output $Y_{1}$} & \multicolumn{5}{|c|}{ Outpu $Y_{\|}$} \\
\hline & TFP & QN & $P G X$ & SE & TAE & TFP & QN & PGX & SE & TAE \\
\hline BR & 0.0104 & 0.0102 & 0.0127 & -0.0083 & -0.0042 & 0.0291 & 0.0052 & 0.0147 & -0.0015 & 0.0109 \\
\hline CD & -0.0241 & 0.0007 & 0.0074 & -0.0309 & -0.0013 & 0.0203 & 0.0001 & 0.0100 & -0.0074 & 0.0176 \\
\hline CFF & 0.0278 & -0.0019 & 0.0181 & 0.0124 & -0.0008 & 0.0417 & 0.0011 & 0.0165 & 0.0046 & 0.0195 \\
\hline CFL & 0.0155 & 0.0041 & 0.0204 & -0.0151 & 0.0062 & 0.0230 & 0.0013 & 0.0162 & 0.0009 & 0.0046 \\
\hline $\mathrm{CH}$ & 0.0027 & -0.0279 & 0.0231 & -0.0148 & 0.0224 & -0.0059 & -0.0065 & 0.0125 & -0.0086 & -0.0033 \\
\hline CIE & 0.0163 & 0.0023 & 0.0150 & 0.0005 & -0.0016 & 0.0460 & 0.0012 & 0.0122 & 0.0085 & 0.0241 \\
\hline $\mathrm{CP}$ & 0.0291 & 0.0045 & 0.0168 & 0.0197 & -0.0120 & 0.0259 & 0.0029 & 0.0127 & 0.0053 & 0.0051 \\
\hline CP/REFER * & 0.0938 & 0.0841 & 0.0142 & -0.0112 & 0.0068 & 0.0369 & 0.0499 & 0.0136 & -0.0241 & -0.0025 \\
\hline DB & 0.0279 & 0.0184 & 0.0183 & -0.0039 & -0.0049 & 0.0419 & 0.0090 & 0.0158 & 0.0010 & 0.0160 \\
\hline DB AG & 0.0601 & 0.0113 & 0.0114 & 0.0140 & 0.0233 & 0.0145 & 0.0045 & 0.0139 & 0.0049 & -0.0089 \\
\hline DSB & 0.0194 & -0.0006 & 0.0165 & 0.0029 & 0.0007 & 0.0220 & -0.0008 & 0.0147 & 0.0034 & 0.0046 \\
\hline EVR & -0.0541 & -0.0063 & 0.0049 & -0.0521 & -0.0006 & 0.0182 & 0.0007 & 0.0064 & 0.0057 & 0.0054 \\
\hline FS & 0.0175 & 0.0094 & 0.0123 & 0.0069 & -0.0110 & 0.0266 & 0.0049 & 0.0136 & 0.0018 & 0.0063 \\
\hline MAV & -0.0221 & -0.0018 & 0.0096 & -0.0183 & -0.0116 & 0.0104 & -0.0003 & 0.0110 & -0.0061 & 0.0057 \\
\hline NS & 0.0238 & 0.0031 & 0.0193 & 0.0001 & 0.0014 & 0.0289 & 0.0010 & 0.0178 & -0.0026 & 0.0126 \\
\hline NS B.V./NS N.V. * & 0.0467 & 0.0028 & 0.0076 & 0.0380 & -0.0017 & -0.0484 & 0.0017 & 0.0148 & -0.0606 & -0.0042 \\
\hline NSB & 0.0264 & 0.0024 & 0.0224 & 0.0042 & -0.0027 & 0.0251 & 0.0013 & 0.0144 & 0.0013 & 0.0082 \\
\hline ÖBB & 0.0225 & 0.0023 & 0.0121 & 0.0089 & -0.0008 & 0.0265 & 0.0010 & 0.0128 & 0.0070 & 0.0057 \\
\hline PKP & -0.0557 & 0.0062 & 0.0068 & -0.0341 & -0.0345 & 0.0370 & 0.0044 & 0.0107 & -0.0082 & 0.0302 \\
\hline RENFE & 0.0317 & 0.0132 & 0.0158 & 0.0070 & -0.0043 & 0.0311 & 0.0065 & 0.0139 & 0.0039 & 0.0068 \\
\hline SJ & 0.0410 & 0.0038 & 0.0223 & 0.0143 & 0.0005 & 0.0316 & 0.0013 & 0.0155 & 0.0000 & 0.0148 \\
\hline SJ/BV & 0.0266 & 0.0182 & 0.0139 & -0.0055 & 0.0000 & 0.0239 & 0.0102 & 0.0135 & -0.0015 & 0.0018 \\
\hline SNCB & 0.0247 & 0.0085 & 0.0137 & 0.0024 & 0.0002 & 0.0278 & 0.0046 & 0.0150 & 0.0008 & 0.0075 \\
\hline SNCF & 0.0236 & 0.0133 & 0.0144 & 0.0033 & -0.0074 & 0.0263 & 0.0048 & 0.0142 & 0.0018 & 0.0056 \\
\hline SNCF/RFF * & 0.0018 & 0.0030 & 0.0090 & -0.0071 & -0.0031 & 0.0309 & 0.0016 & 0.0128 & 0.0063 & 0.0101 \\
\hline SZ & 0.0465 & -0.0001 & 0.0099 & 0.0093 & 0.0274 & 0.0420 & 0.0000 & 0.0112 & -0.0012 & 0.0320 \\
\hline TCDD & -0.0108 & -0.0072 & 0.0093 & 0.0009 & -0.0138 & 0.0024 & -0.0017 & 0.0084 & 0.0003 & -0.0046 \\
\hline VR & 0.0354 & 0.0019 & 0.0209 & 0.0165 & -0.0040 & 0.0107 & 0.0003 & 0.0140 & -0.0013 & -0.0023 \\
\hline VR/RHK * & 0.0353 & 0.0011 & 0.0158 & 0.0178 & 0.0006 & 0.0272 & 0.0015 & 0.0131 & 0.0136 & -0.0010 \\
\hline ZSR & 0.0131 & -0.0004 & 0.0044 & -0.0346 & 0.0437 & 0.1040 & -0.0001 & 0.0092 & -0.0020 & 0.0969 \\
\hline Average & 0.0184 & 0.0059 & 0.0139 & -0.0019 & 0.0004 & 0.0259 & 0.0037 & 0.0132 & -0.0018 & 0.0108 \\
\hline
\end{tabular}

* firms with less than 3 observations

Changes in scale seem to have had little effect on TFP growth (around $-0.18 \%$ in both output set measures) ${ }^{17}$. These results are in conformity with those obtained by Gathon and Pestieau (1995) and Sánchez and Villarroya (2000), who also found that the greater part of the growth in the level of TFP is explained by technological progress and to much lesser degree by changes in efficiency and scale economies. 
Using the output measured by passenger (tonne)-kilometres, the companies with the highest levels of total productivity growth rate are DB AG, SZ, SJ and VR, while EVR, CD and TCDD show the lowest performance levels in the sample. If instead, we analyse total productivity growth rate using train-kilometres as output, CIE, SZ, DB, CFF and PKP are those with the highest rate of total productivity growth, and $\mathrm{CH}$ and TCDD are those with the lowest levels. Note, that companies such as CD, CIE, EVR, MAV, ZSR and specially PKP appear to have significantly higher performances when output is measured by train-kilometres than passenger (tonne)-kilometres. Additionally we can observe that with the enlargement of DB (from DB to DB AG) there was a significant reduction in the productivity growth rates when output set is measured in train-kilometres but, when output passenger (tonne) - kilometres is used, a significant increase in total productivity rates is observed. For companies which have implemented vertical separation of infrastructure management and operations we also find significant changes in productivity performance.

\section{Conclusions}

This paper has examined the economic performance of a panel of twenty-seven European railways over the period 1972 and 1999. The cost structure of the railway industry was analysed using a stochastic frontier cost function with associated cost share equations modelled within the framework of a translog cost system.

Our analysis indicates that European railways experience a significant cost increase due to allocative inefficiency behaviour, with a sample mean increase in variable costs due to allocative inefficiency of around 20\%. Regarding technical inefficiency, we find a mean increase in variable costs of around $3.6 \%$. The firms which show the highest levels of inefficiencies are those that are 
large in size and that have invested more in new technology over the last decades. A comparison of our results with those obtained in Parisio (1999), also using a cost frontier approach, shows a high degree of similarity. Further comparisons with other study results are made difficult due to the use of dissimilar methodology and data.

\begin{abstract}
Despite the difficulty in making comparisons of allocative and technical inefficiencies with previous studies, our results are consistent in 'global' terms with those of previous studies in identifying relatively cost efficient (NS, CFF, SJ, SNCF and RENFE) and cost inefficient (TCDD and FS) firms. Perhaps the most important point of departure from the previous European railway literature is our finding that allocative inefficiency plays a much more significant rule in generating cost increases than technical inefficiency. The conclusion is that firms experience greater difficulty in finding correct input proportion than in achieving equiproportionate excess input usage.
\end{abstract}

Regarding policy options for cost inefficiencies, it seems that the impact of subsidies on efficiency is very dependent of the types of subsidy and the way in which they are provided. The separation of infrastructure and operations can lead to an increase in technical inefficiency, but it also seems to allow for a betterment adjustment of inputs used and revenue outputs, which in aggregate actually result in efficiency cost savings of around 5\%. Inefficiencies related to excess service capacity arise more from passenger service than freight service, revealing the greater difficulties which European railway firms face in making adjustments in passenger output supply. Overall, we observe that inefficiencies can essentially be explained by the excess of capacity supplied and by the discrepancies resulting from an over-employment of labour input. 
Our analysis of TFP confirms the results obtained by Gathon and Pestieau (1995) and Sánchez and Villarroya (2000), that it is technological progress, and not variation in efficiency levels, that provides the most convincing explanation for variance in TFP growth. Scale economies do not appear important in explaining the growth of TFP, but our results do confirm embodied technological progress (such as high-speed technology and quality of service improvements) as an important element favouring productivity. Thus, in the present context of increasing public sector de-regulation and liberalisation, it may be important to ensure that infrastructure investments and modernisation are encouraged in order to avoid decreasing levels of productivity over time.

\section{References}

Andrikopoulos, A. A. and J. Loizides (1998). "Cost structure and productivity growth in European railway systems." Applied Economics 30: 1625-1639.

Bauer, P. W. (1990a). "Decomposing TFP growth in the presence of cost inefficiency, nonconstant returns to scale, and technological progress." The Journal of Productivity Analysis 1: 287-299.

Bauer, P. W. (1990b). "Recent developments in the econometric estimation of frontiers." Journal of Econometrics 46: 29-56.

Bognetti, G. and R. Fazioli (1999). "Liberalization problems and prospects in European railways." Annals of Public and Cooperative Economics 70(2): 303-318. 
Bosco, B. (1996). "Excess-input expenditure estimated by means of an input-distance function: the case of public railways." Applied Economics 28: 491-497.

\begin{abstract}
Braeutigam, R. R., A. F. Daughety and M. Turnquist (1984). "A firm specific analysis of economies of density in the U.S. industry." The Journal of Industry Economics XXXIII(1): 3-20.
\end{abstract}

Brinkhoff, T. (2002). The Principal Agglomerations of the World. www.citypopulation.de.

Brown, B. W. and M. B. Walker (1995). "Stochastic specification in random production models of cost-minimizing firms." Journal of Econometrics 66: 175-205.

Coelli, T. and S. Perelman (2000). "Technical efficiency of European railways: a distance function approach." Applied Economics 32: 1967-1976.

Couto, A. (2005). The impact of high speed technology on demand and productivity in European railways: an econometric analysis. Faculty of Engineering of the University of Porto. unpublished $\mathrm{PhD}$ dissertation.

ECIS (1996). The State of European Infrastructure, European Centre for Infrastructure Studies.

Farrell, M. J. (1957). "The measurement of productive efficiency." Journal of the Royal Statistical Society Series A: 253-281. 
Friedlaender, A. F., E. R. Berndt, J. S. W. Chiang, M. Showalter and C. A. Vellturo (1993). "Rail cost and capital adjustments in a quasi-regulated environment." Journal of Transport Economics and Policy 21: 131-152.

Gathon, H. J. and S. Perelman (1992). "Measuring technical efficiency in European railways: a panel data approach." The Journal of Productivity Analysis 3: 135-151.

Gathon, H. J. and P. Pestieau (1995). "Decomposition efficiency into its managerial and its regulatory components: the case of European railways." European Journal of Operational Research 80: $500-507$.

Hunter, C. H. (1992). "Growth accounting when technical change is embodied in capital." American Economic Review 82 (4): 964-980.

IMF (2001). International Financial Statistics. www.imf.org, International Monetary Fund.

Jane's (1995). Jane's World Railways - Railway Systems. London, Ed. James Abbott.

\author{
Jowdrow, J. A., C. A. K. Lovell, I. V. Materov and P. Schmidt (1982). "On the estimation of \\ technical inefficiency in the stochastic frontier production function model." Journal of \\ Econometrics 19: 233-238.
}


Klein, L. (1962). An introduction to econometrics, Englewood Cliffs: Prentice-Hall.

\begin{abstract}
Kumbhakar, S. (1991). "The measurement and decomposition of cost -inefficiency: the translog cost system." Oxford Economic Papers 43: 667-683.
\end{abstract}

\begin{abstract}
Kumbhakar, S. C. (1988a). "Estimation of input-specific technical and allocative inefficiency in stochastic frontier models." Oxford Economic Papers 40: 535-549.
\end{abstract}

\begin{abstract}
Kumbhakar, S. C. (1988b). "On the estimation of technical and allocative inefficiency using stochastic frontier functions; the case of U.S. class 1 railroads." International Economic Review 2(4): 727-743.
\end{abstract}
Kumbhakar, S. C. (1989). "Economic performance of U.S. class I railroads: a stochastic frontier approach." Applied Economics 21: 1433-1446.

Loizides, J. and E. G. Tsionas (2002). "Productivity growth in European railways: a new approach." Transportation Research Part A 36(A): 633-644.

Maps.com (1999). World Elevation. www.maps.com. 
Morrison, C. J. (1985). "Primal and dual capacity utilisation: An application to productivity measurement in the U.S. automobile industry." Journal of Business \& Economic Statistic 3(4): 312324.

Morrison, C. J. (1986). "Productivity measurement with non-static expectations and varying capacity utilization." Journal of Econometrics 33: 51-74.

NationalGeographic and ESRI (2002). ArcAtlas: Our Earth - snow cover. www.nationalgeographic.com, ESRI.

Nelson, R. A. (1984). "Regulatism, capital vintage, and technical change in the electric utility industry." Review of Economics and Statistics LXVI(1): 59-69.

OECD (2002). OECD Financial Statistics (www.oecd.org/dataoecd).

OECD-Statistics (2001a). Measuring capital - OECD Manual: Measurement of capital stocks, consumption of fixed capital and capital services. (www.SourceOECD.org): 1-132.

OECD-Statistics (2001b). Measuring capital - OECD Manual: Measurement of capital stocks, consumption of fixed capital and capital services. (www.SourceOECD.org): 1-132. 
OECD-Statistics (March-2001). Measuring productivity-OECD Manual: Measurement of aggregate and Industrial-level productivity growth. www.SourceOECD.org. Paris: 1-154.

Oum, T. H., M. W. Tretheway and Y. Zhang (1991). "A note on capacity utilization and
measurement of scale economies." Journal of Business \& Economic Statistic 9(1): 119-123.

Oum, T. H. and Y. Zhang (1991). "Utilisation of quasi-fixed inputs and estimation of cost functions - an application to airline costs." Journal of Transport Economics and Policy 25: 121-138.

Parisio, L. (1999). "A comparative analysis of European railroad efficiency: a cost frontier approach." Applied Economics 31: 815-823.

Perelman, S. (1986). "Frontier défficacite et performance technique des chemins de fer." Annales de l'économie publique, sociale et coopérative $\left(n^{\circ} 4\right)$ : 445-459.

Preston, J. (1994). Does size matter? A case study of Western European railways. UTSG

Conference, Institute for transport Studies, University of Leeds.

Sánchez, P. C. and J. M. Villarroya (2000). "Efficiency, Technical change and productivity in the European rail sector: a stochastic frontier approach." International Journal of Transport Economics XXVII(1): 55-76. 
Schmidt, P. and R. C. Sickles (1984). "Production frontier and panel data." Journal of Business \& Economic Statistics 2: 367-374.

Uden, M. v. (2001). The European Railway Stock Lists, The European Railway Server (http://mercurio.iet.unipi.it).

UIC (1972-1999). International Railway Statistics. Paris, International Union of Railways.

Vellturo, C. A., E. R. Berndt, A. F. Frielaender, J. S. W. Chiang and M. H. Showalter (1992).

"Deregulation, mergers, and cost savings in Class I U.S. railroads, 1974-1986." Journal of Economics \& Management Strategy 1(2): 339-369.

World Bank (2002). World Development Indicators (http://www.worldbank.org/data/). 


\section{Appendix 1: Railway firms}

\begin{tabular}{ll} 
BR & British Railways \\
CFF & Swiss Federal Railways \\
CFL & Luxembourg National Railway Company \\
CIE & Irish Transport Company \\
CP & Portuguese Railway Company \\
DB & German Federal Railway \\
CH & Hellenic Railway Organisation - Greece \\
DSB & Danish State Railways \\
FS & Italian State Railways \\
NS & Netherlands Railways \\
NSB & Norwegian State Railways \\
ÖBB & Austrian Federal Railways \\
RENFE & Spanish National Railway System \\
SJ & Swedish State Railway \\
SNCB & Belgian National Railway Company \\
SNCF & French National Railway Company \\
VR & Finish State Railways \\
TCDD & Turkish Republic State Railways \\
BC & Belarus Railways \\
BDZ & Bulgarian State Railways \\
CD & Czech Republic Railways \\
CFR & Railways of Romania \\
EVR & Estonian Railways \\
MAV & Hungary State Railways \\
PKP & Polish State Railways \\
SZ & Slovenian Railways \\
ZSR & Slovak Republic Railways \\
\hline
\end{tabular}


${ }^{1}$ The short-run perspective is justified by the fact that the railways operate in a heavy regulated environment where decisions on the economic adjustment of "way and structures" capital factor cannot be based on static cost minimisation criteria. For this reason, a quasi-fixed capital factor is introduced in the model as an exogenous variable.

${ }^{2}$ As Bauer (1990b) notes, unfortunately, these estimates cannot be shown to be consistent estimates of $\operatorname{lnC}_{\tau}$, since the variability of the conditional distribution $\operatorname{lnC}_{\tau}$ given $\zeta$ is independent of the sample size (i.e., $\zeta$ contains only imperfect information about $\ln \mathrm{C}_{\tau}$ ).

${ }^{3}$ See Couto (2005) for full descriptions and treatments of these variables. The choice of variables was very constrained by the unavailability of reliable variable measures for the whole period.

${ }^{4}$ Due to a lack of quality data over the whole period and for all firms, cross-section data is unbalanced with yearly observations covering the whole period for some firms but other firms with reduced number of observations, mainly those from the Eastern countries. For this reason, and attending that the reliability of the data for these countries, corresponding to a period of political and economic system transition, can be questioned in the presence of high levels of inflation, any conclusions relative to these country companies should be taking with cautions.

${ }^{5}$ The traditional application of the perpetual inventory method requires the direct estimation of depreciation from which the net capital stock is obtained indirectly. The alternative approach, which was used in this study, is to use the age price profiles to directly estimate the net capital stock from which depreciation is obtained indirectly. This alternative method has the advantage of all stock data being necessarily consistent with each other because the age-efficiency profiles (used to estimate the productive capital stock) determine the age-price profiles (used to estimate the net capital stock and depreciation).

${ }^{6}$ Investments expenditures were obtained by first differencing the reported un-depreciated value of stocks between each year. Due to the lack of some year/firm's observations or misleading capital stock observations reported in UIC data, investment expenditures for these years and firms were obtained from figures reported in ECIS (1996) and firm annual reports. Additionally, for companies with several capital stock reavaluations or accounting changes in the period, the firm's revaluation capital stock and the following year observations were interpreted as new firm observations.

${ }^{7}$ The hyperbolic age-efficiency profile has been calculated by a function of the form, $h_{\tau}=\frac{T-(\tau-1)}{T-\beta(\tau-1)}$, where T, the duration of the asset, was set at 50 years and $\beta$ was set 0.75 according to the values given in OECD-Statistics (2001b) for infrastructure assets. The function assumed for retirement patterns, $F_{t}$, was the simultaneous exit function, which means that any investment produced at a certain point in time will be completely discarded at a certain future time Railway infrastructures have, as a physical characteristics, their long life duration which means that infrastructures have the capacity of being able to produce until they reach their service life and are then discarded/abandoned from service, which usually happens in block (i.e. there is a reduced number of infrastructures which have the ability of being partially discarded and still continuing in service; nevertheless, even in the cases where this is possible, due to its long life duration, this would occur far from the period in study, more than 30 years). Productive capital stocks were converted in constant 1995 US\$ through out the use of PPP's 1995 deflators. 
${ }^{8}$ The average life service for railway transport equipment considered in this study was twenty five years. A delayed linear retirement pattern was used as the distribution function $F_{\mathrm{t}}$ characterising discards occurrences, and the hyperbolic age-efficiency profile $h_{\mathrm{t}}$ was calculated assuming $\mathrm{T}=30$ years and $\mathrm{b}=0.5$, the value used by ABS and BLS for most types of machinery and equipment.

${ }^{9}$ The rate of depreciation was calculated as the ratio between the real depreciation $\left(D_{\mathrm{t}}\right)$ and the real net capital stock $\left(K_{\mathrm{t}}\right)$ at constant prices. $D \mathrm{t}$ is the real depreciation term, which is given by: $D_{t}=\sum_{\tau}\left(z_{\tau} F_{\tau}-z_{\tau+1} F_{\tau+1}\right) \frac{I N_{t-\tau-1}}{q_{t-\tau-1}}$, where $I N_{\mathrm{t}}$ is the nominal investment expenditure on the asset at time $t$, for $\mathrm{q}_{\mathrm{t}}$ (the price index deflator for the asset of age zero in year t) the GDP deflator was used, the age-price profile $\left(Z_{s}\right)$ was derived from the age-efficiency profile $\left(h_{s}\right)$ and the retirement profile $\left(F_{s}\right)$ and assuming a "real discount rate" of $4 \%$ (as usual in empirical applications).

${ }^{10}$ Since these alternative options to increase passenger rail traffic velocity are very distinct in technology as well as in the magnitude of investment expenses, two distinct dummy variables were introduced, one which detects the presence of high-speed technology which allows running vehicles with maximum speed above $220 \mathrm{KM} / \mathrm{h}$, labelled DHS, and the other dummy variable which assumes the value one when firms own titling trains vehicles and the value zero otherwise, this dummy variable was labelled DTT. To capture the effect on costs of expanding investment on high speed technology besides the dummy variables described above, two more variables HS220 and TT were introduced, which were defined as the product of initial dummy variables by the respective logarithm of the number of vehicles existent in each technology. This way it is possible to analyse the effect of the increment in this type of technologies in costs and consequently in productivity.

${ }^{11}$ All models gave elasticities of substitution with similar values for the majority of observations, however, in some models for a restricted number of data points the figures of these elasticities are extremely high in comparison with the remaining observations, which consequently yielded, for these models, higher values of standard deviations.

${ }^{12}$ Since the test hypotheses of no-autocorrelation and homoskedastic regression, as well as the test against cross group correlation, were rejected at the $1 \%$ of probability level, the computation was modelled so allowing for groupwise heteroskedasticity, cross group correlation and within group autocorrelation. Since the basic command for TSCD in Limdep programming demand a balanced panel with contiguous group data, all firms without complete data set (twenty eight observations) were excluded from the regression; thus the number of observations in this groupwise regression models were reduced to 364 observations.

${ }^{13}$ It is usual in transport analysis to express economies of scale in two distinct concepts: economies of network size and economies of density. However, we will present only the estimation relating to economies of density in order to have conformity with the subsequent treatment for estimation of TFP growth.

${ }^{14}$ Having estimated the short-run variable cost function, the short-run disequilibrium total cost function is obtained by adding the parcel of capital input cost $\left(T C=g\left(Y, W_{v}, Z, K, t\right)+r . K\right)$ where $\mathrm{r}$ is the rental price of the capital stock $\mathrm{K}$. By differentiating this equation with respect to capital stock, equilibrium capital stock is obtained when the opportunity cost of capital (the price of capital r) equals the firm's shadow value of capital, and consequently, the optimal level of 
capital stock is that which results from this equality. Examples of this approach to short and long-run costs can be found in Braeutigam et al. (1984), Vellturo et al. (1992) and Friedlaender et al. (1993).

${ }^{15}$ Similar estimation of economies of network size showed, for the European railways which make up the sample, slight increasing returns to scale.

${ }^{16}$ The variables which compose the vector of the service quality and network characteristics $\left(q_{m}\right)$ are: the quality of service variable (qvmax), the network length (NET) and the high speed technology variables (DHS, DTT, HS220, TT)

17 The average value is negative which means that scale has had a decreasing effect on total productivity growth. However, if instead of the average of mean firm values we calculate the mean value of all observations in the sample we obtain an average positive value around of $0.1 \%$. 